\title{
A generative-oriented model-driven design environment for customizable video surveillance systems
}

\author{
Nuno Cardoso ${ }^{*}$, Pedro Rodrigues ${ }^{1}$, João Vale ${ }^{1}$, Paulo Garcia ${ }^{1}$, Paulo Cardoso ${ }^{1}$, João Monteiro1, \\ Jorge Cabral ${ }^{1}$, José Mendes ${ }^{1}$, Mongkol Ekpanyapong ${ }^{2}$ and Adriano Tavares ${ }^{1}$
}

\begin{abstract}
To tackle the growing complexity and huge demand for tailored domestic video surveillance systems along with a high demanding time-to-market expectation, engineers at IW Automação, LDA a are exploiting video surveillance domain as families of systems that can be developed following a pay-as-you-go fashion rather than developing an exnihilo new product. Several and different new functionalities are required for each new product's hardware platforms (e.g., ranging from mobile phone, PDA to desktop PC) and operating systems (e.g., flavors of Linux, Windows and MAC OS X). Some of these functionalities have special economical constraints of development time and memory footprint. To better accommodate all the above listing requirements, a model-driven generative software development paradigm supported by mainstream tools is proposed to offer a significant leverage in hiding commonalities and configuring variabilities across families of video surveillance products while maintaining the new product quality.
\end{abstract}

\section{Introduction}

Nowadays there is a growing demand for video surveillance systems [1]. The development of these systems is increasingly complex since there is a high demand for new products with a rising number of different requirements [2].

However, it can be noticed that the increasing complexity is induced by the variability in tasks related to image capturing, image processing, communications and computer vision [3]. Furthermore, it is now expected that the system runs in different hardware platforms, ranging from desktop PCs to low cost embedded boards, mobile phones, etc.

The majority of system designers use modular architectures based on Microsoft DirectShow implementation $[4,5]$ in order to address the growing complexity of novel products and the ever increasing time-to-market pressure. In this implementation, all system functionalities are implemented as individual plug-ins or filters. Later, these plug-ins will be connected following the pipeline

\footnotetext{
*Correspondence: linun77@gmail.com

1 Department of Industrial Electronics, Centro Algoritmi, University of Minho, Braga, Portugal

Full list of author information is available at the end of the article
}

execution model (filter graph) through a generic interface. In this way, higher flexibility, customizability and reusability can be achieved for video surveillance oriented design. Furthermore, at run time, only the required plug-ins for a given configuration will be loaded and a new system configuration can be implemented by simply defining new plug-ins to be loaded and the connections among them.

In the case of embedded systems, such an implementation incurs a huge abstraction penalty [6-9], since it is based on weighty language's dialects (i.e., features that increase overhead in space and performance), like dynamic polymorphism.

In order to tackle these embedded systems constraints [6], video surveillance systems can be implemented as a software product line (SPL) $[2,10]$ by designing plug-ins which fit all possible video surveillance system's configurations and simply reusing the common features of several configurations while considering the variable features that identify each system specific configuration.

Based on practical experience at IVV in tackling the design challenges of video surveillance systems, new software technologies such as model driven development [11] and generative programming [12], should be

\section{黑 Springer}


combined with SPL engineering to bridge the abstraction gap between domain modeling and feature modeling.

The remainder of this article is structured as follows. The following section deliberates related works. Next, in Section 3, Microsoft DirectShow is reviewed, together with brief discussion on how to reverse engineer it. Section 4 focuses on video surveillance domain engineering by implementing the feature modeling. Section 5 presents the details of video surveillance application engineering, showing how model-driven engineering techniques are employed to establish the relationships between video surveillance feature models and video surveillance domain model. It also describes how offline and online partial evaluators [13] will be applied, respectively, at whole system and filter designs. In Section 6, the implementation of a SDK for video surveillance product line development is described. Section 7 presents some results. Finally, in Section 8 we conclude the article and present some directions of future study.

\section{Related work}

The amount of research works in this field is very large, so this short literature survey describes related works that apply similar software technologies, describes video surveillance systems design and/or manages variability.

In [2], a mixture of model-driven and generative programming techniques are proposed to support variability configuration of video surveillance systems. In order to better manage features combination at runtime, it separates the variability in domain and code representation spaces.

In [14], Aspect-Oriented Programming (AOP) is employed in order to avoid crosscutting concerns by managing the existing variability in operating systems domain. Even though the use of AOP enables higher levels of granularity when compared with other variability management strategies, it incurs in higher overhead depending on the applied type of advice and the inability to manage variability in systems with several instances of the same class each with different requirements.

In [15], to better tackle the time-to-market pressure during the developing of digital games the use of generative programming was proposed. In [16], a Fujaba plug-in for SPL development was described using model-driven techniques. The main focus is on how to bridge the gap between feature modeling and domain modeling with annotations.

In [17], new mechanisms are explored in order to better specify and understand the relationship between system variability and requirements by combining SPL, Aspectorientation and model-driven techniques. The Ample project, presents two complementary languages, RDL and VML4RE, to allow specifying and reasoning about crosscutting relationships between features and requirements.
In [18], Matilda is described. Matilda is a model-driven development framework that allows automatic code generation through transformations using UML models while addresses the abstraction gap and lack of traceability related to current model-driven development practice. In this way, the developer analyses, designs and executes applications in modeling layer, abstracting it from the programming layer.

In [19], generative programming as an automatic selection and assembly of components based on configuration knowledge and a set of construction rules is proposed. A layered architecture, called GenVoca is used and the configuration knowledge is implemented using $\mathrm{C}++$ template metaprogramming as generator.

\section{Microsoft directshow and its reverse engineering}

DirectShow is a pipeline-based media-streaming architecture and API based on component object model (COM) framework that provides a common interface for media across several programming languages. It's a filter-based framework that implements a complex multimedia application by connecting several filters of three main kinds (i.e., source, transform and renderer filters) through their input and output pins using filters graphs to provide specific functionality [5].

Since DirectShow SDK only offers partial code of the framework, we run a reverse engineering process using IDA Pro [20] on quartz.dll to figure out its real architecture, starting with the CFilterGraph class.

Due to the limited space, we'll only present how the implemented interfaces at CFilterGraph class were identified as well as the overhead of a filter class like CMjpegDec. Listing 1 partially shows the assembly code of the CFilterGraph constructor where the initialization of the virtual tables for each defined interface is visible. The register esi points to the data member of the class (i.e., this pointer) while the first memory addresses point to the interfaces virtual tables implemented by CFilterGraph class. Figure 1 presents CFilterGraph class layout with special focus on its thirteen direct and ten indirect interfaces through the pointer, $m_{p} p F G C o n t r o l$, to an object of CFGControl class that encapsulates all filter graph control functionalities.

\subsection{Listing 1 Partial Assembly code of CFilterGraph class constructor \\ 1 ??0CFilterGraph@@AE@PAGPAUIUnknown@@- PAJ@Z proc near \\ 2 \\ 3 ... mov esi, ecx ; esi $=$ this... call \\ 4. CBaseFilter::CBaseFilter(ushort const $*$,IUnknown $*$,CCritSec $*$,_GUID \\ 5 const \&) push [ebp+cbData] mov dword ptr [ebx], offset}




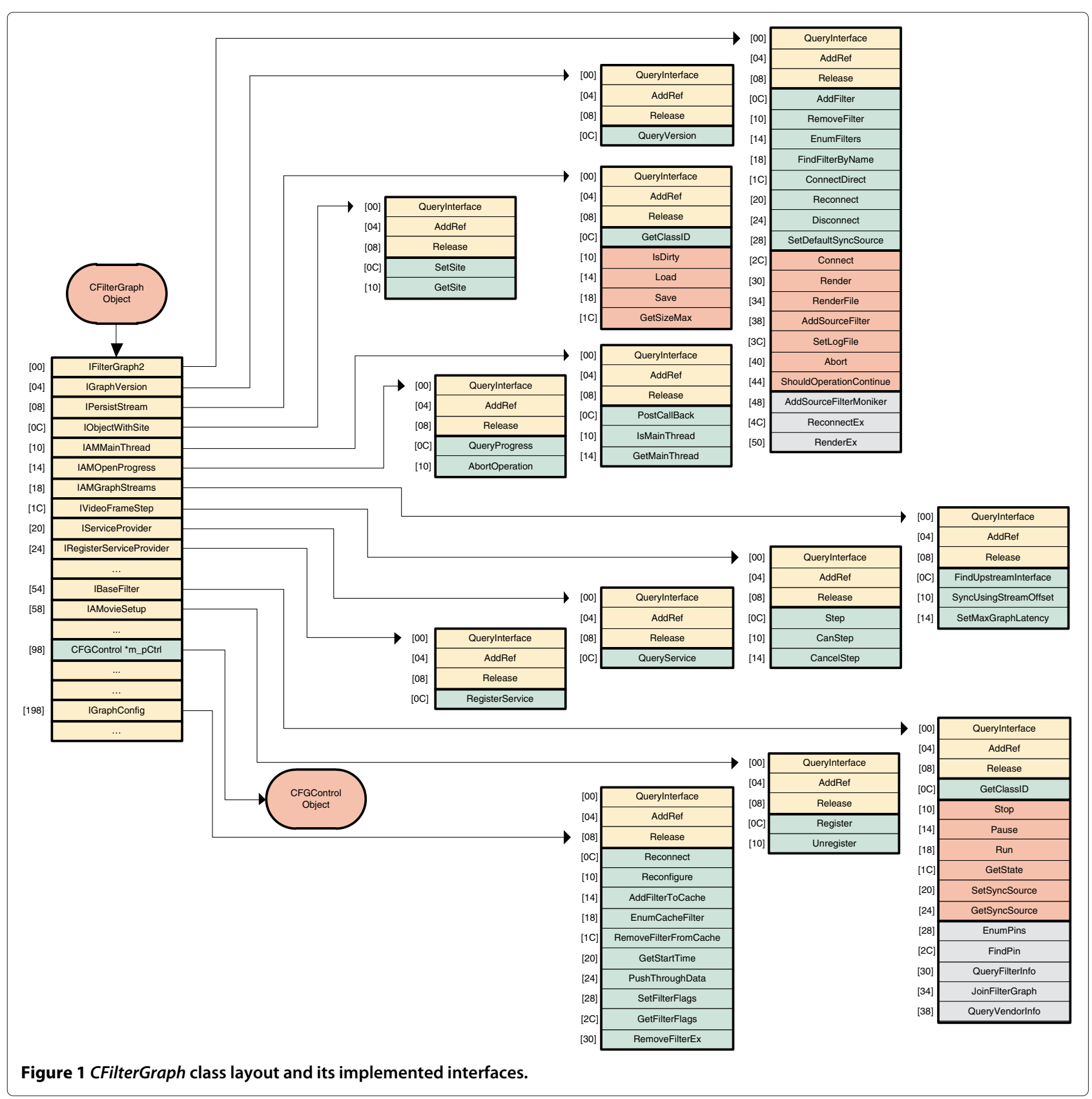

6 CFilterGraph::'vftable'\{for 'CUnknown'\} xor ebx, ebx lea ecx,

7 [esi+9Ch] mov dword ptr [esi], offset

8 CFilterGraph::'vftable' ffor 'IFilterGraph2'\} mov dword ptr

9 [esi+4], offset CFilterGraph::'vftable'\{for 'IGraphVersion'\} mov

10 dword ptr [esi+8], offset CFilterGraph::'vftable'\{for

11 'IPersistStream'] mov dword ptr [esi+0Ch], offset

12 CFilterGraph::'vftable'\{for 'IObjectWithSite'\} mov dword ptr
13 [esi+10h], offset CFilterGraph::'vftable'\{for 'IAMMain Thread'\} mov

14 dword ptr [esi $+14 h]$, offset CFilterGraph:: vftable' ffor

15 'IAMOpenProgress' $\}$ mov dword ptr [esi+18h], offset

16 CFilterGraph::'vftable' ffor 'IAMGraphStreams'\} mov dword ptr

17 [esi+1Ch], offset CFilterGraph::'vftable'\{for 'IVideoFrameStep'\} mov

18 dword ptr [edi], offset CFilterGraph::'vftable' $\{$ for 
19 'IServiceProvider' mov dword ptr [esi+24h], offset

20 CFilterGraph::'vftable'\{for 'IRegisterServiceProvider'\} mov dword

21 ptr [esi+54h], offset CFilterGraph::'vftable' for 'IBaseFilter' $\}$ mov

22 dword ptr [esi $+58 \mathrm{~h}]$, offset CFilterGraph::'vftable'\{for

23 'IAMovieSetup'\} mov [esi+98h], ebx...

From the CMjpegDec class constructor analysis, Listing 2, the following code segments were identified, informing us that it inherits from CVideoTransformFilter class and implements IUnknown, IBaseFilter and IAMovieSetup interfaces:

i. Initialization of the stack frame from line 1 to 3 ;

ii. Calling of CVideoTransformFilter constructor as a base class from line 6 to 10 ;

iii. Registering of virtual tables addresses for each implemented interface from line 15 to 17 ;

iv. Initialization of class variable members from line 18 to 24 ;

v. Returning to the caller with stack restoring from line 25 to 31 .

3.2 Listing 2 CMjpegDec class constructor assembly code

1 mov edi, edi push ebp ; save ebp mov ebp, esp ;

2 save stack frame push esi ; save esi push edi;

3 save edi push offset _CLSID_MjpegDec ; push CLSID_MjpegDec

4. (REFCLSID) push [ebp+arg_4] ; push pUnk (LPUNKNOWN) mov

5 esi, ecx ; esi = this push [ebp+arg_O] ; push pName (TCHAR

$\left.6^{*}\right)$ call

CVideoTransformFilter::CVideoTransformFilter(...) ; call

7 the base class xor edi, edi ; edi $=0$ push edi

8 ; push nDefault (INT) push offset aMjpegnoskip ; push

9 “MJPEGNoSkip” (LPCTSTR lpKeyName) push offset AppName ; push

10 "Quartz" (LPCTSTR lpAppName) mov dword ptr [esi], offset

11 CMjpegDec::'vftable'\{for 'CUnknown'\} mov dword ptr $[\mathbf{e s i}+0 \mathrm{Ch}]$,

12 offset CMjpegDec::'vftable' \{for 'IBaseFilter'\} mov dword ptr

13 [esi+10h], offset CMpegAudioCodec::'vftable' for 'IAMovieSetup' mov

14 [esi+0COh], edi mov [esi+0C $8 \mathrm{~h}]$, edi mov [esi+0CCh], edi mov

15 [esi+0DOh], edi mov [esi+0D4h], edi call

16 GetProfileIntW( $(\mathrm{x}, \mathrm{x}, \mathrm{x})$; GetProfileInt("Quartz", "MJPEGNoSkip", 0);
$17 \operatorname{mov}[\mathbf{e s i}+0 \mathrm{~B} 0 \mathrm{~h}]$, eax mov eax, [ebp+arg_8]; eax = phr;

18 (HRESULT $*$ phr) mov [eax], edi ; $*$ phr $=$ NOERROR; pop

19 edi ; restore edi mov eax, esi ; ret = this pop

20 esi ; restore esi pop ebp ; restore ebp retn

$210 \mathbf{C h}$; return and clean stack

Listing 3 shows the assembly code of CMjpegDec::Transform method that receives a media sample (i.e., IMediaSample "pIn) and outputs another media sample (i.e., IMediaSample " $p \mathrm{Out}$ ), with the image decompression carried out at line 7 by the function DecompressBegin that receives as arguments an output $\mathrm{YCbCr}$ buffer, an input JPEG image buffer and an image header info.

\subsection{Listing 3 CMjpegDec::Transform method partial assembly code \\ 1 mov [ebp+ms_exc.disabled], 1 push dword ptr [esi $+0 \mathrm{COh}]$ call \\ 2 DDecompressEnd@4; DecompressEnd(x)push [ebp+pInfoHeader] ; \\ 3 image header push edi ; image src buffer push \\ 4 dword ptr [esi+0COh] ; image dst buffer call _DecompressBegin@12 \\ 5 ; DecompressBegin $(x, X, X)$; decompress frame or \\ 6 [ebp+ms_exc.disabled], OFFFFFFFFh and [ebp+var_6C], 0}

Listing 3 shows the assembly code of CMjpegDec::Transform method that receives a media sample (i.e., IMediaSample $*$ pIn) and outputs another media sample (i.e., IMediaSample $*$ pOut), with the image decompression carried out at line 7 by the function DecompressBegin that receives as arguments an output $\mathrm{YCbCr}$ buffer, an input JPEG image buffer and an image header info.

Listing 4 partially shows the disassembling of DecompressBegin function indicating that MJpegDec filter uses IJG JPEG library during decompression process. Deeper analysis of this function revealed through the $C$ routines into the library that it's a generic JPEG decompressor implementation full of variations and consequently with a severe dead code overhead.

\subsection{Listing 4 Partial disassembling of DecompressBegin function \\ 1 lea edi, [esi $+1 F 8 h]$ lea eax, [esi +8$]$ push eax call \\ 2 _jpeg_exception_error@4; ;peg_exception_error $(x)$ mov [edi], eax \\ 3 push edi call _jpeg_create_decompress@4 ; \\ 4 jpeg_create_decompress $(\mathrm{x})$ mov byte ptr [esi+399h], 1 mov \\ 5 eax, $[\mathbf{e b x}+10 \mathrm{~h}]$ test eax, eax jnz loc_74823996}


Complementing this information with a reverse engineering on the SDK source code, Figures 2, 3 and 4 describe the DirectShow static architecture.

\section{Video surveillance domain engineering}

We'll follow the usual approach for SPL development organized in domain engineering and application engineering, with the former also defined as "development for reuse" and split into domain analysis, domain design and domain implementation. Application engineering is also defined as "development with reuse". SPL technology consists of a set of tools, methods and techniques to create a set of products, software systems, from a common base for all products (i.e., all system settings) [13]. Furthermore, to bridge the gap between variability model and model elements and automate the mapping of system configuration to the domain model and generating the final code, we followed the MDD process proposed in [16] as showing in Figure 5.

\subsection{The domain analysis}

The domain analysis is split in domain scope that determines which systems and features are part of video surveillance domain (Figure 6) and feature modeling which identifies the commonality and variability as well as the relationship among features in the variability model. Feature modeling was proposed as a part of the Feature
Oriented Domain Analysis (FODA) [21], and since then, it is applied in various fields. The model features are visually represented by functionality diagrams that model system variability notwithstanding the used implementation mechanism, such as inheritance, templates or conditional compilation.

For the video surveillance domain, several feature diagrams are provided-one for the filter graph or pipeline and one for each type of filter (Figures 7, 8, 9 and 10).

The feature diagram of the filter graph, Figure 7, identifies and classifies all filters that compose the filter graph as well as all possible links between them. The root node represents the concept (i.e., filter graph) that consists of three features, one for each type of filters that make up the filter graph (i.e., source, transform and renderer filters). The source filter concept contains two alternative features, live device or file, meaning that in any video surveillance system instance, only one of them should be included. The transform filter concept consists of two orfeatures (i.e., codecs and Processing), meaning that any video surveillance system instance has at least one of them.

As indicated by the cardinalities [0..*] and [1..*], the transform filters are optional and a filter graph can present none, one or several of them, and source and rendering filters are mandatory, i.e., a filter graph or pipeline must present at least one of each.

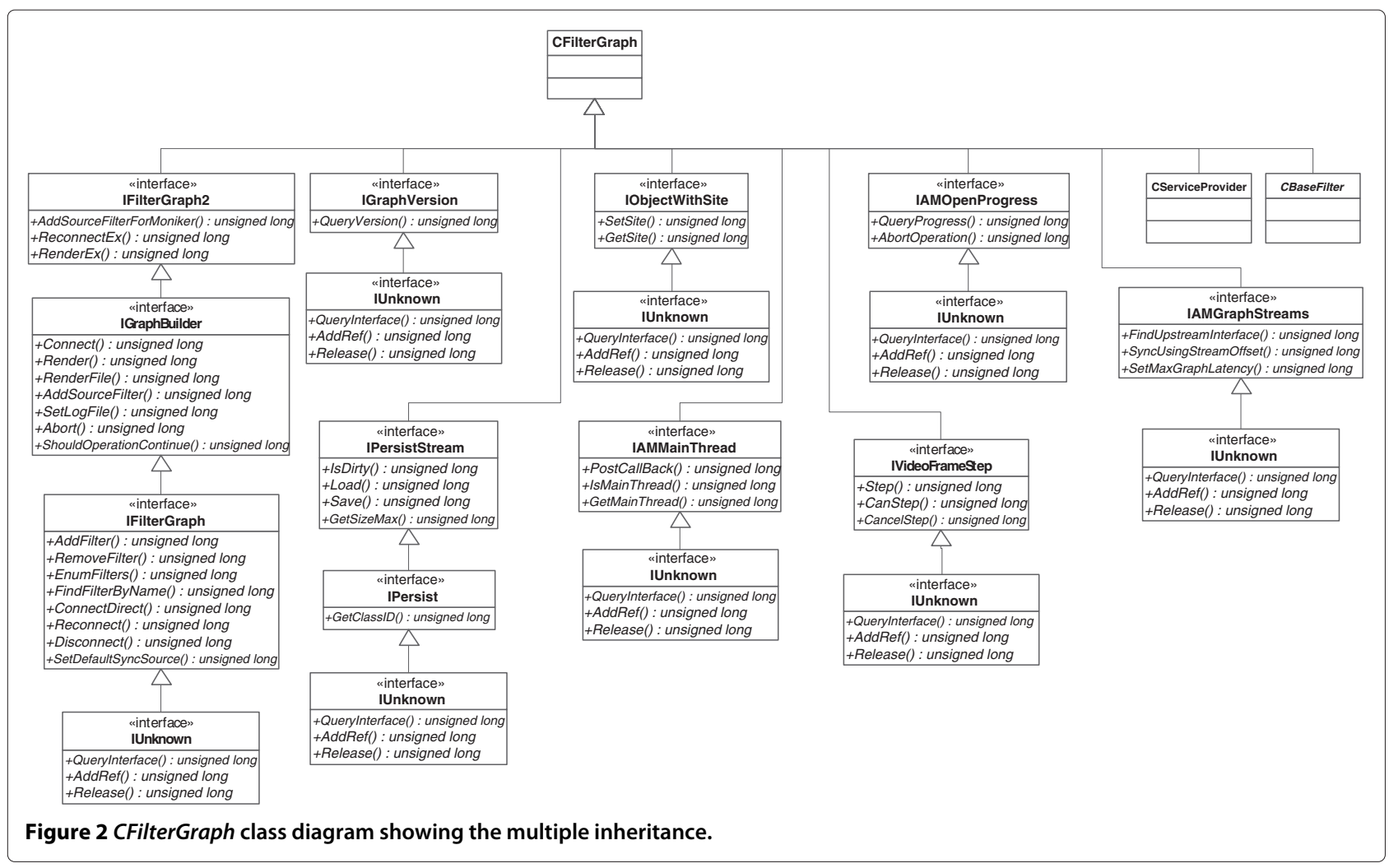




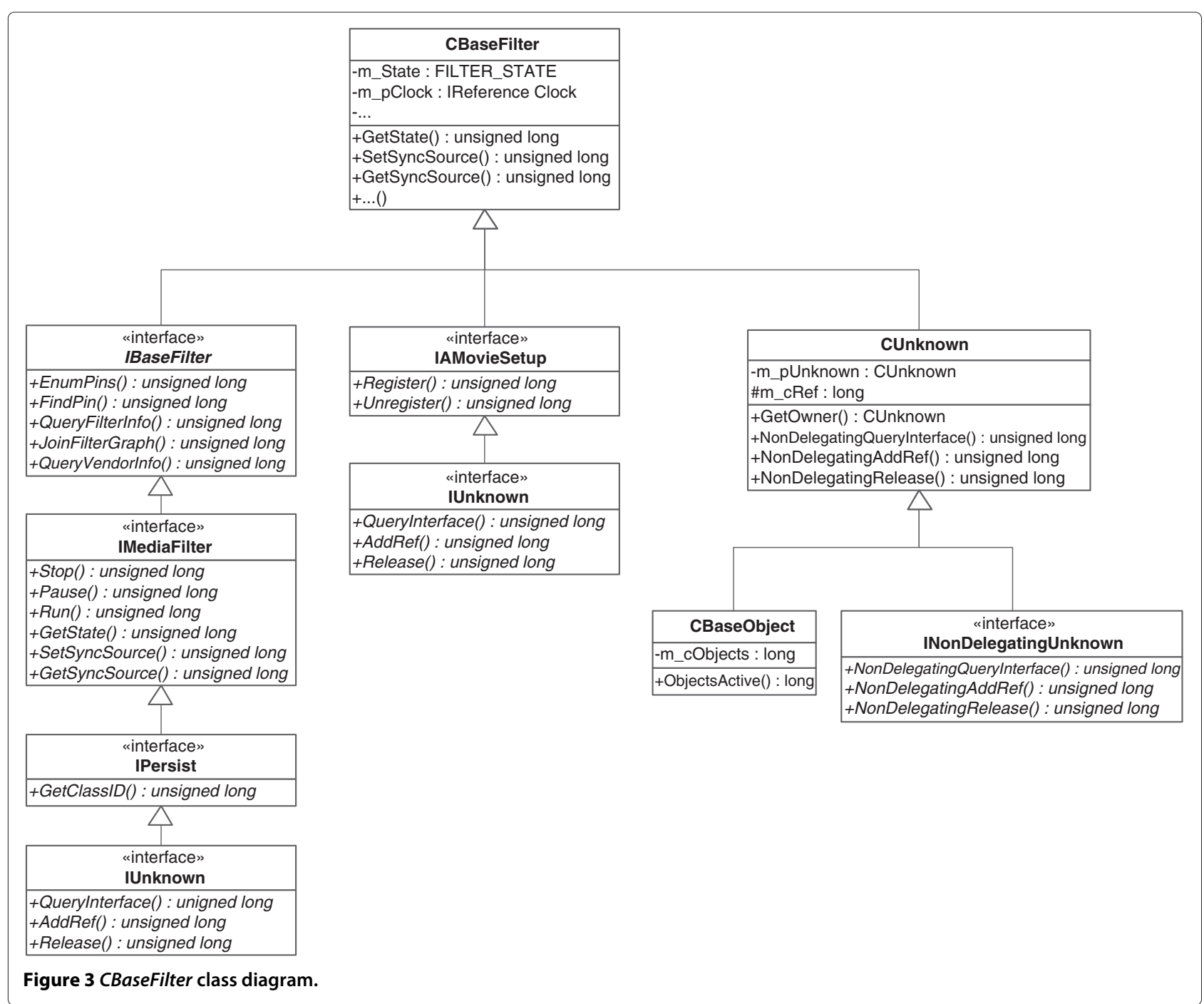

All the above feature diagrams are internally represented using XML notation as can be shown in Listings 5 and 6 for the description of the File renderer filter and filter graph, respectively.

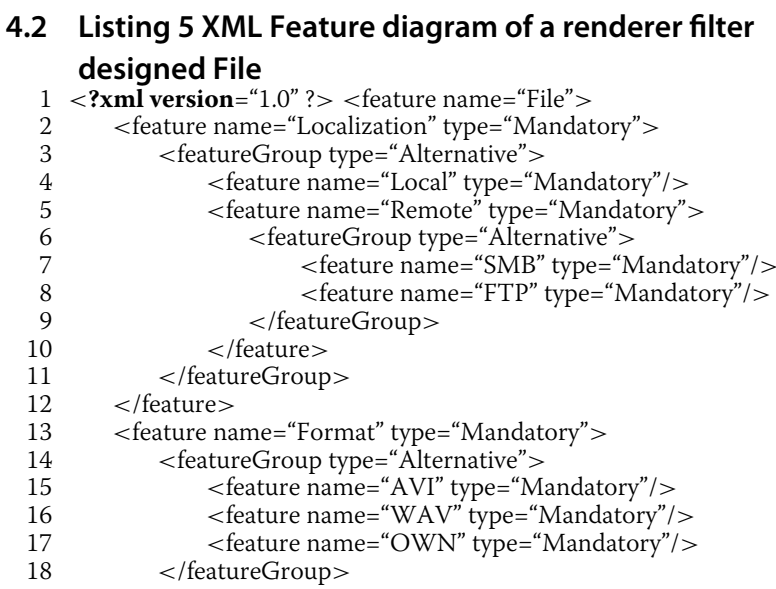

4.2 Listing 5 XML Feature diagram of a renderer filter designed File

$1<$ ?xml version="1.0" ? > <feature name="File" $>$

$<$ feature name="Localization" type="Mandatory" $>$ $<$ featureGroup type="Alternative" $>$

$<$ feature name="Local" type="Mandatory" $\mid>$

$<$ feature name $=$ "Remote" type $=$ "Mandatory" $>$ $<$ featureGroup type="Alternative" $>$ $<$ feature name="SMB" type="Mandatory"/> $<$ feature name="FTP" type="Mandatory" $\mid>$ $</$ featureGroup $>$ $</$ feature $>$

$</$ featureGroup $>$

$</$ feature $>$

$<$ feature name="Format" type $=$ "Mandatory" $>$ $<$ featureGroup type="Alternative" $>$

$<$ feature name="AVI" type="Mandatory" $\mid>$

$<$ feature name="WAV" type $=$ "Mandatory" $/>$

$<$ feature name $=$ "OWN" type $=$ "Mandatory" $/>$

$<$ /featureGroup $>$

4.3 Listing 6 Filter graph XML Feature diagram

$1<$ ?xml version="1.0" ?> < feature name="Filter Graph" > $<$ feature min="1" $\max =$ "*" name="Source Filter"type="Mandatory" $>$ $<$ featureGroup type $=$ "Alternative" $>$

$<$ feature name="Live Device" type $=$ "Mandatory" $>$

$<$ featureGroup type="Alternative" $>$

$<$ feature name="Analogic Camera" type="Mandatory"|>

$<$ feature name $=$ "Microphone" type $=$ "Mandatory"/>

$<$ feature name="IP Camera" type="Mandatory"/ $>$

$<$ /featureGroup $>$

$<$ /feature $>$

$<$ featureGroup $>$

$</$ feature $>$

..

$<$ feature $\min =" 1$ "max=" $*$ "name="Renderer Filter" type="Mandatory" $>$

$<$ featureGroup type $=$ "Or" $>$

$<$ feature name="Transmission" type $=$ "Mandatory" $>$

$<$ featureGroup type $=$ "Alternative" $>$

$<$ feature name="HTTP" type $=$ "Mandatory" $/>$

$<$ feature name $=$ "RTSP" type $=$ "Mandatory" $\mid>$

$<$ /featureGroup $>$

$<$ /feature $>$

$<$ /featureGroup $>$ 


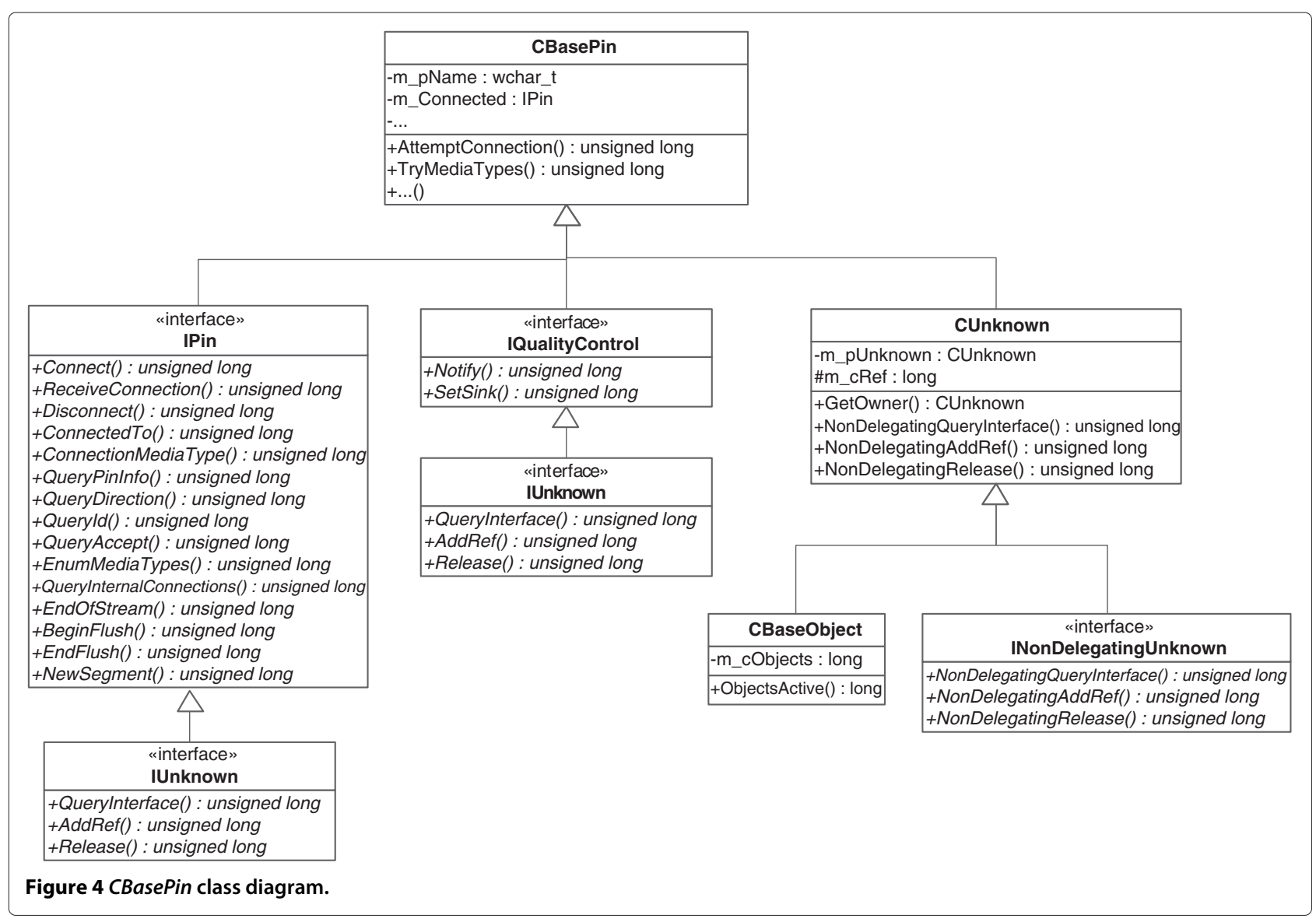

$25</$ feature $>$

$26</$ feature $>$

\subsection{The domain design and implementation}

Domain design is the next step after the specification of applications and components in video surveillance domain and its main goal is developing a common architecture for the video surveillance family of products. Having defined the common architecture, the implementation domain proposes developing configurable and customizable components to support the implementation of the video surveillance system domain architecture [22]. The implemented generative-friendly framework for video surveillance software product family, basically, handles variability at two levels of granularity (intra-filter and inter-filter) and consists of:

(i) Several and different artifact templates encoded in $\mathrm{C}++$ template metaprogramming for each type of filter that is generated;

(ii) A filter graph artifact template describing the pipeline as a confederation of filters based on component technology and encoded in $\mathrm{C}++$ template metaprogramming;

(iii) A set of meta-expressions and presence conditions with direct correspondence to the artifact templates and their variability points, and expressed in terms of features used to map features in feature models to the artifact templates, and so, giving semantics to those features [23];

(iv) A set of implicit rules for filters connection and validation, are internal and implicitly defined as part of filter graph within its built-in Intelligent Connector. These rules are also encoded in $\mathrm{C}++$ metaprogramming artifact templates.

\subsubsection{The filter artifact template}

The filter artifact template does not implement an artifact template for each filter type, in opposition to other filter implementations that classify each filter by category using specific classes for the purpose. Instead, all the filters follow the same implementation that allows the representation of artifact filters in a more flexible manner. This methodology was used to allow easier refactoring of legacy code from external libraries that implements some filters functionalities. The framework allows exploring the granularity at two levels: coarse-grained and fine-grained. A coarse-grained granularity allows changing between filters; as an example, changing a JPEG transformation filter by a H.264 transformation filter. A fine-grained granularity allows having different instances of the same 


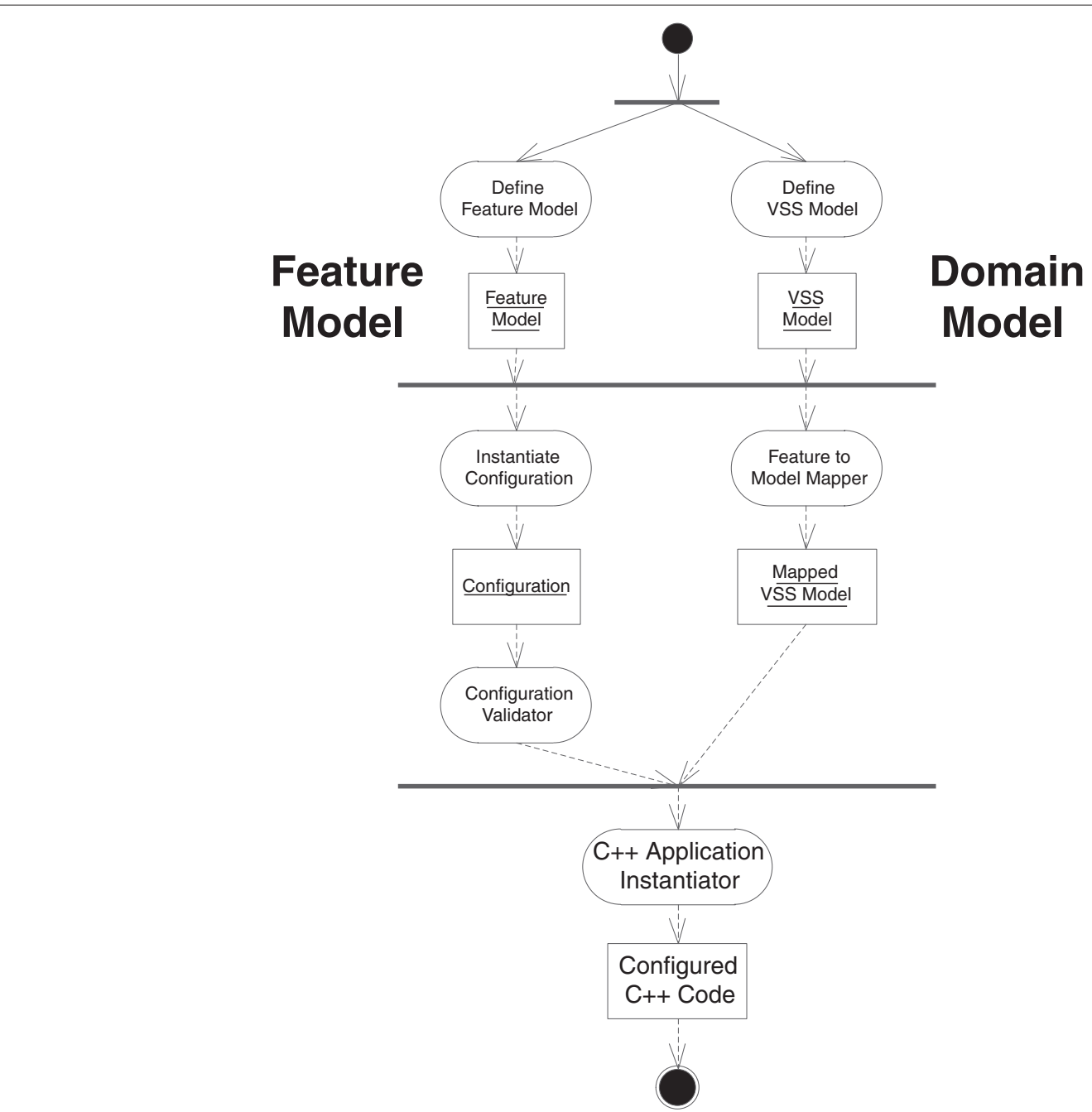

Figure 5 MDD process which combines Feature models and domain models in VSS.

filter to satisfy the system requirements, e.g., the JPEG transformation filter can have a generic implementation and an optimized implementation.

To develop a new filter artifact template, the unique requisites that the filter needs to implement are:

(i) Define the data type named type that informs what is the type of the filter (source, transform, renderer);

(ii) The Initialize and Cleanup methods that initialize and cleanup all the filter resources;

(iii) The Connect method that allows connecting other filter to this filter;

(iv) The Start, Stop and Pause methods that control the execution data flow;

(iv) The Process method that process data.

Most of the artifact templates related to different kind of filters are automatically generated running an online partial evaluator on legacy codes from previous video surveillance projects (external libraries). Such refactoring process was implemented based on an automatic partial evaluation for the $\mathrm{C}++$ language named transformer [13] on a first stage and then the output was adapted to our filter template architecture.

In the following sub-section, two filter artifact implementations are presented, one for an input filter and the other for a transformation filter.

\subsubsection{The input filter artifact template}

Now let's take the input filter V4L2 as represented by the feature diagram in Figure 8 to partially describe the artifact template of such kind of filters. Due to huge extension of the code we'll focus on the filter Cleanup method needed to free all allocated resource by the filter. Three created structures, one for each capture method and a skeleton artifact template for CV4L2Source along with the Cleanup method are created as shown by Listings 7 and 8 , respectively. 

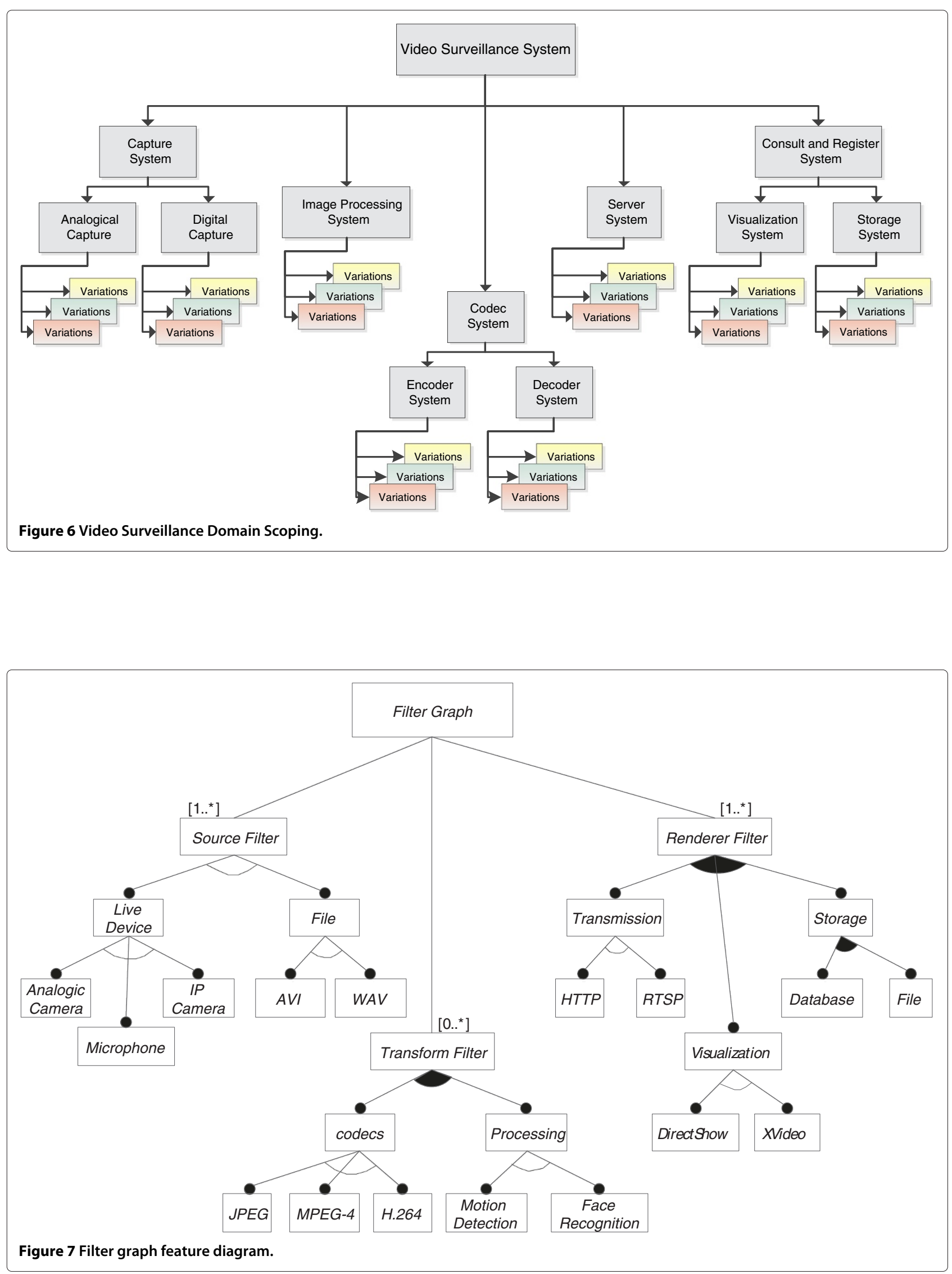


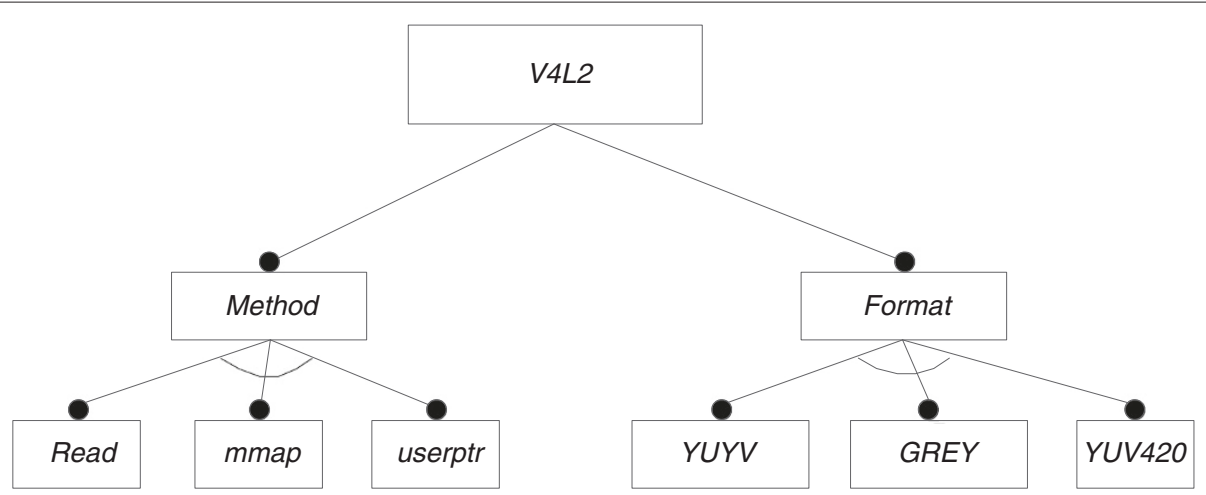

Figure 8 Feature diagram of a V4L2 source filter.

\subsection{Listing 7 Data type definition for the capture mode}

$1 / /$ read method

2 struct $v 412 \_$read_method_t \{\} ;

3

4. // mmap method

5 struct v412_mmap_method_t \{\} ;

6

7 // userptr

8 struct v4l2_userptr_method_t \{\} ;

\subsection{Listing 8 CV4L2 class skeleton artifact template code \\ 1 template $<$ typename IOMethod $>$ \\ 2 class CV4L2Source \{ \\ 3 public: \\ 4 // defines the filter type \\ 5 typedef source_filter_t type; \\ 6 \\ $7 \quad$ CV4L2Source() \{\}}

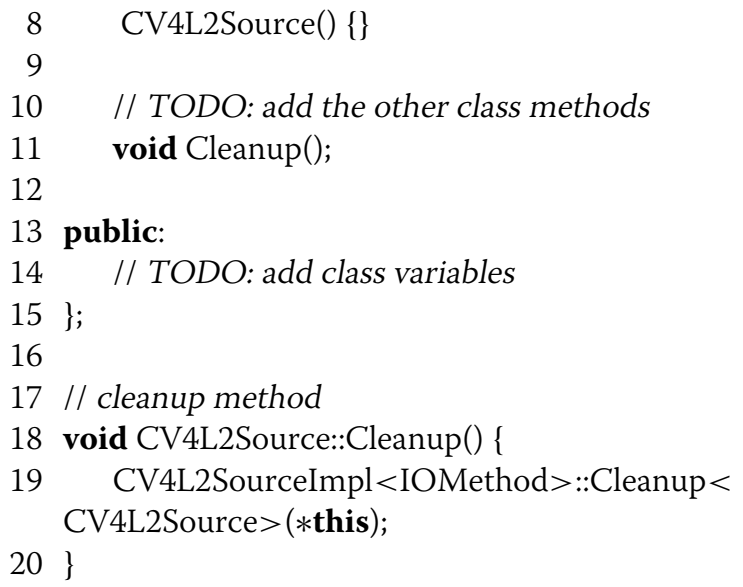

The Cleanup method uses a template data structure, CV4L2SourceImpl (see Listing 9), which defines three specialized templates, one for each capture mode. Based on the selected capture mode, one of these templates

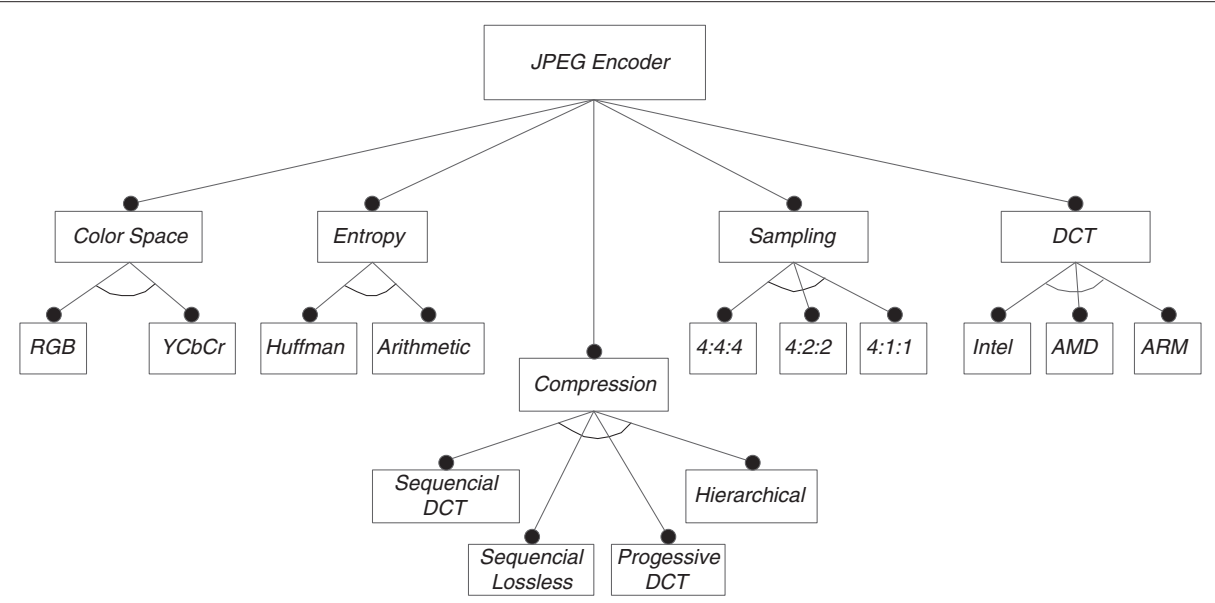

Figure 9 Feature diagram of a transform filter for JPEG decompression. 


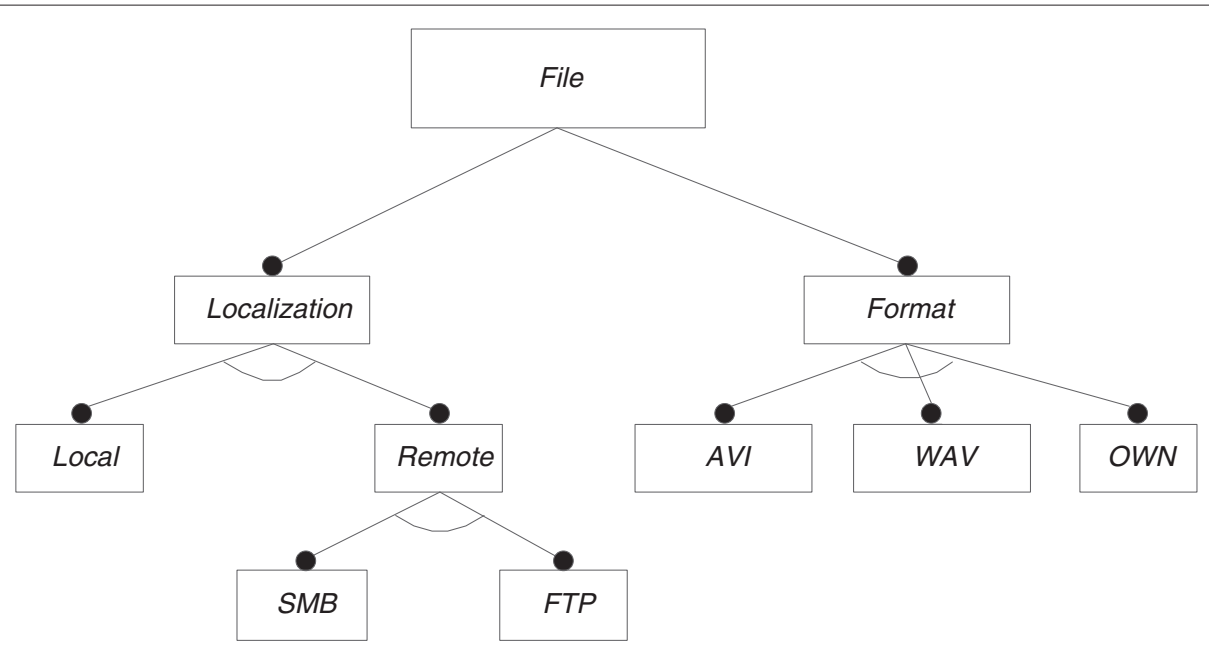

Figure 10 Graphical Feature diagram of a renderer filter designed File.

is instantiated with an inline Cleanup function whose body is statically patched into the place where Cleanup is supposed to be called.

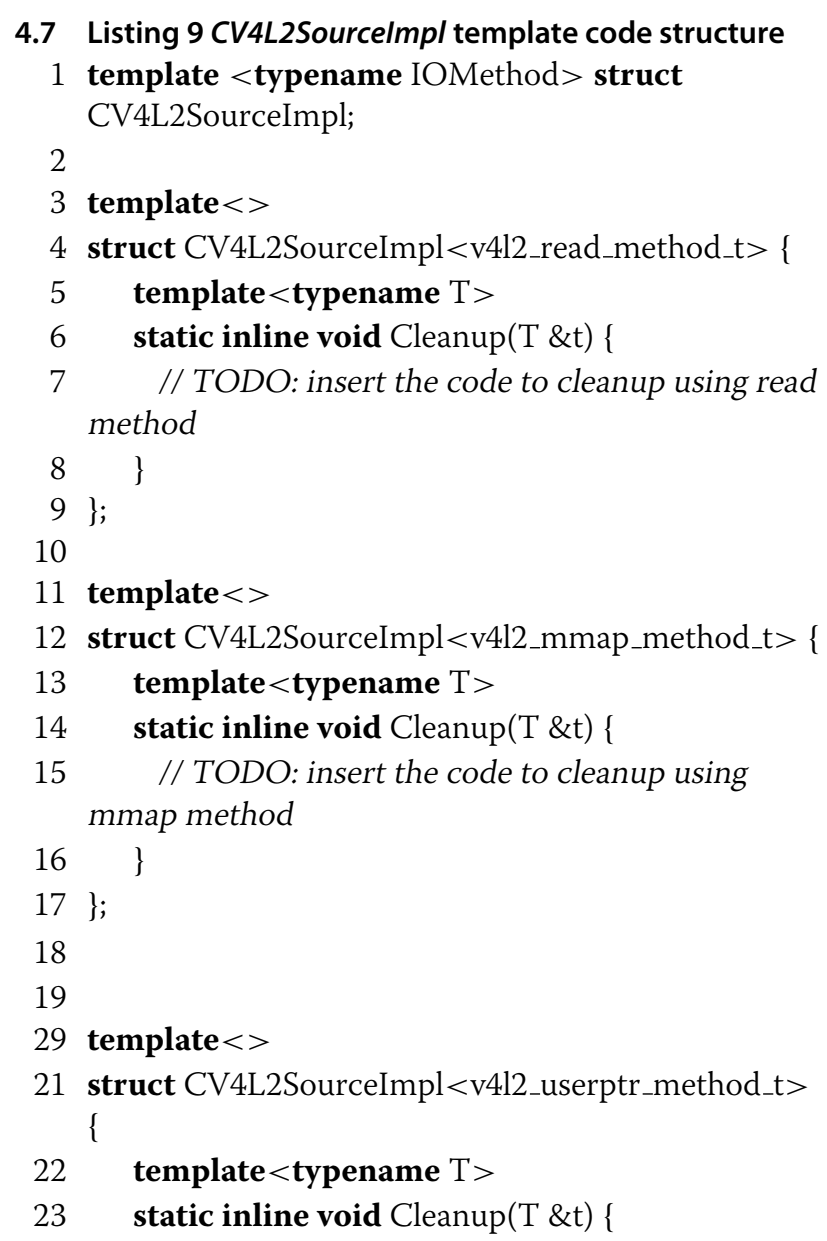

24 // TODO: insert the code to cleanup using userptr method

25

$26\}$

Finally, a concrete instance of the CV4L2Source artifact template using one of the capture modes (i.e., read_method) is given in Listing 10 .

\subsection{Listing 10 Instantiation of CV4L2Source class with read capture mode \\ 1 CV4L2Source $<$ v4l2_read_method_t $>$ v4l2; \\ 2 V4l2.Cleanup();}

\subsubsection{The transform filter artifact template}

Taking the diagram in Figure 9 let's partially describe the artifact template related to the concept JPEG Decoder that decompresses images streaming in JPEG format to raw format (e.g., YCbCr or RGB). Again due to the code huge extension we'll only discuss the implementation of sampling and color Space features. Three data type will be defined, one for each JPEG's sampling, 4:4:4. 4:2:2 and 4:1:1 (Listing 11) as well as their associated specialized template structure CJPEGSamplingImpl as shown in Listing 12.

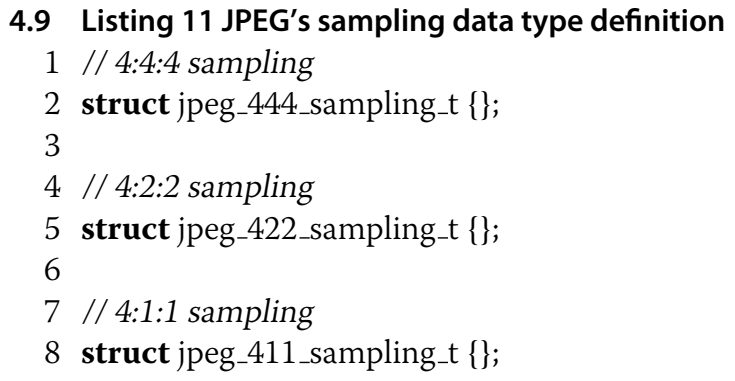




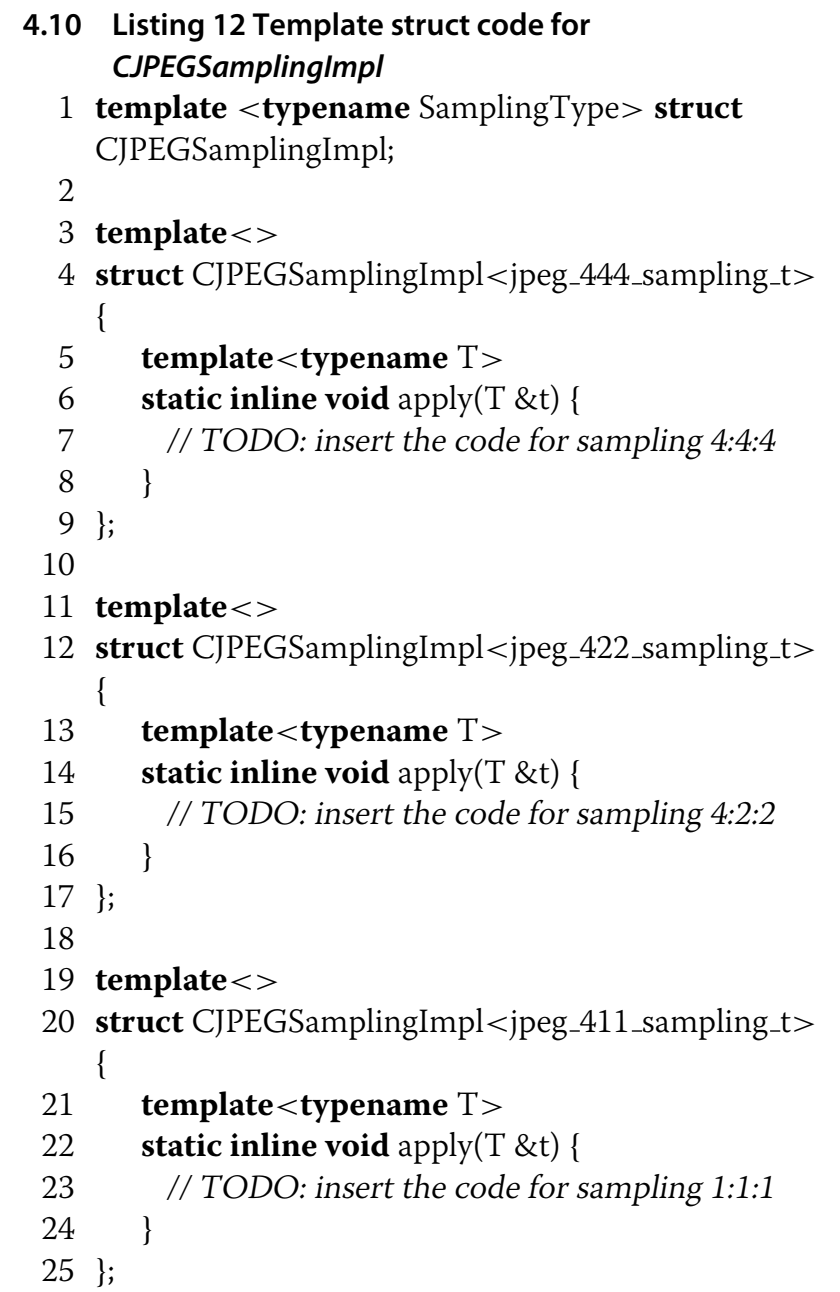

Each specialized template defines an inline function apply that implements the related sampling functionality. Upon instantiation, only one of the specialized templates is instantiated by the compiler, depending on the type of data SamplingType. The Color Space concept implementation follows similar steps (Listings 13 and 14) but as it includes alternative features, $\mathrm{YCbCr}$ and $\mathrm{RGB}$, two different types of data should be created as illustrated in Listing 13.

\subsection{Listing 13 Definition of data types for JPEG's Color implementation \\ $1 / /$ YCbCr color space \\ 2 struct jpeg_ycbcr_colorspace_t \{\} ; \\ 3 \\ 4 // RGB color space \\ 5 struct jpeg_rgb_colorspace_t \{\} ;}

\subsection{Listing 14 CJPEGColorSpacelmp/ template structure code}

1 template $<$ typename ColorSpaceType $>$ struct

CJPEGColorSpaceImpl;
2

3 template $<>$

4 struct

CJPEGColorSpaceImpl $<$ jpeg_ycbcr_colorspace_t $>\{$

template $<$ typename $\mathrm{T}>$

static inline void apply $(\mathrm{T} \& \mathrm{t})\{$

// TODO: insert the code for color space $\mathrm{YCbCr}$ \} \}

10

11 template $<>$

12 struct

CJPEGColorSpaceImpl $<$ jpeg_rgb_colorspace_t $>\{$

13 template $<$ typename $\mathrm{T}>$

14 static inline void apply $(\mathrm{T} \& \mathrm{t})\{$

15 // TODO: insert the code for color space RGB

$16\}$

17 \};

Now the CJPEGDec artifact template can be presented (see Listing 15) and should be later instantiated with two template input parameters given by the data types of SamplingType and Color Space features. CJPEGDec::Decode( ) takes as input parameter a buffer containing the image information stored in JPEG format. In Listing 16 sampling 4:4:4 and RGB color space are chosen.

\subsection{Listing 15 CJPEGDec is the template artifact related to JPEG Decoder concept}

1 template $<$ typename SamplingType, typename ColorSpaceType $>$

2 class CJPEGDec

3\{

4 public:

$5 \quad / /$ defines the filter type

6 typedef transform filter_t type;

7

8 CJPEGDec() \{\}

$9 \sim \mathrm{CJPEGDec}()\{\}$

// TODO: add the other class methods

void Decode(CBuffer $*$ pBuffer);

public:

// TODO: add class variables

7 \};

18

19 // decode method

20 template $<$ typename SamplingType, typename ColorSpaceType $>$

21 void CJPEGDec::Decode(CBuffer $*$ pBuffer)

22\{ 


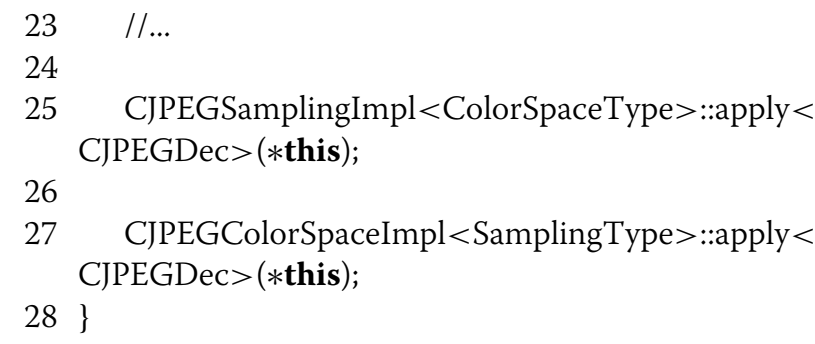

\subsection{Listing 16 Instantiation example of the artifact} template CJPEGDec

1 // image buffer

2 CBuffer buffer;

3

4. CJPEGDec $<$ jpeg_444_sampling_t, jpeg_rgb_colorspace_t $>$ dec;

5 dec.Decode(\&buffer);

\subsubsection{The filter graph artifact template}

To implement the CFilterGraph class, specialized templates are used to instantiate an appropriate artifact template which creates an instance of filter graph with a predefined number of filters. The CFilterGraph artifact template receives a Boost MPL $[24,25]$ vector with the data types of all filters and the number of filters as its input parameters. The number and data type of each filter depends on the configuration required to implement the desired functionality and the simplest filter graph configuration contains at least two types of filters: a source filter and a renderer filter (see Listing 17).

\subsection{Listing 17 Creating a CFilterGraph instance using a data type, Types, representing a vector with 2 filters: CV4L2Source and CXVRenderer \\ 1 typedef mpl::vector $<$ CV4L2Source, CXVRenderer > Types; \\ 2 CFilterGraph < Types, mpl::size $<$ Types $>$ ::value $>$ fg;}

By default nineteen specialized templates are created, allowing at least the instantiation of a CFilterGraph with at least two and at most twenty filters with the number of specialized templates reconfigured by changing the compilation variable MAX_FILTERGRAPH_SPECIALIZATIONS (see Listing 18).

\subsection{Listing 18 A snippet of CFilterGraph C++ template metaprogramming artifact template \\ 1 template $<$ typename FilterTypes, int $\mathrm{N}>$ class CFilterGraph; \\ 2 \\ 3 template $<$ typename FilterTypes $>$ \\ 4 class CFilterGraph $<$ FilterTypes, $2>\{$ \\ 5 typedef typename mpl::at $<$ FilterTypes, mpl::long_<0 > >::type Filter0;} mpl::long_<1 $>>$ ::type Filter1;

7

8 Filter0_f0;

9 Filter 1 f1;

10 public:

11 Filter0\& const GetFilter(mpl::int_<0>) \{return $f 0 ;\}$

12 Filter $1 \&$ const GetFilter(mpl::int_< $<1>$ ) \{return_f1;

13 \};

14

15 template $<$ typename FilterTypes $>$

16 class CFilterGraph $<$ FilterTypes, $3>\{$

17 typedef typename mpl::at< $<$ FilterTypes, mpl::long $<0>>$ ::type Filter0;

18 typedef typename mpl::at<FilterTypes, mpl::long_<1>>::type Filter1;

19 typedef typename mpl::at<FilterTypes, mpl::long_ $<2>>$ ::type Filter2;

20

21 Filter0f0;

22 Filter1 f1;

23 Filter2f2;

24 public:

25 Filter0\& const GetFilter(mpl::int_<0>) \{return $\mathrm{f} 0$;

26 Filter $1 \&$ const GetFilter(mpl::int_<1>) \{return $f 1 ;$ \}

27 Filter $2 \&$ const GetFilter(mpl::int $<2>$ ) \{return f2;

28 \};

$29 \ldots$

To access each type of filters that make up the filter graph, the CFilterGraph class implements the method GetFilter which receives as input parameter the Boost MPL data type int _ $_{-}$ith the index of the filter and returns the data type of the filter. To simplify and also offer some flexibility to the implementation of each CFilterGraph template specializations, a few macros based on the token past operator are used, such as DEFINE_FILTER_TYPE(n), and CREATE_FILTER_GRAPH(n) to define the data types of each filter at index $n$ in the vector of types and create each specialization with $n$ filters, respectively (Listings 19 and 20.

\subsection{Listing 19 Macro that defines the type of filters: $n$ is the index in the vector of types \\ 1 \#define DEFINE_FILTER_TYPE(n)\ \\ 2 typedef typename mpl::at $<$ TList, mpl::long_<n $>>::$ type Filter\#\#n;}




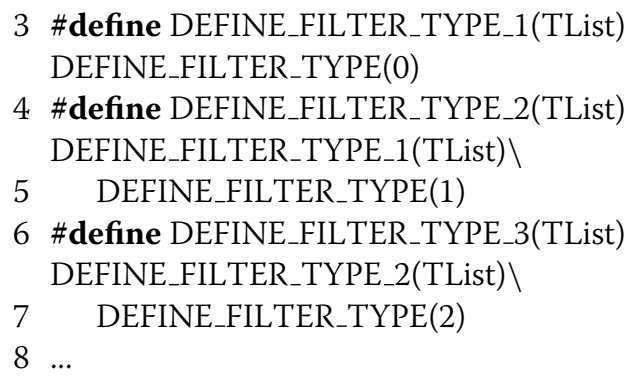

4.18 Listing 20 Macro that creates specialized templates of CFilterGraph: $n$ is the number of filters

1 \#define CREATE_FILTER_GRAPH(n)\

2 template $<$ typename FilterTypes $>1$

3 class CFilterGraph $<$ FilterTypes, $\mathrm{n}>1$

$4 . \quad\{\backslash$

5 DEFINE_FILTER_TYPE_\#\#n(FilterTypes);

6 FILTER_TYPE_\#\#n;

7 public: $\backslash$

8 GET_FILTER_\#\#n;

$9 \quad$;

To create the class variables of each filters and the filter graph GetFilter methods, similar macros are also used and the final implementation of CFilterGraph artifact template will be as shown in Listing 21 .

\subsection{Listing 21 Final implementation of the CFilterGraph artifact template \\ 1 template $<$ typename FilterTypes, int $\mathrm{N}>$ class CFilterGraph; \\ 2 \\ 3 CREATE_FILTER_GRAPH(2); \\ 4. CREATE_FILTER_GRAPH(3); \\ 5 ... \\ 6 CREATE_FILTER_GRAPH(20);}

Listing 22 shows an example of video image capture from an ONVIF compliant IP camera. To capture the images from the IP camera, the input filter RTSP (CRTSPSource) is used, as the IP camera sends images using the JPEG compressed video format, the JPEG transform filter (CJPEGDec) is used and the xvideo renderer filter allows seeing the images on the screen (CXVRenderer. To control and receive camera events a filter that implements web services is used. For that purpose several auxiliary artifact templates were implemented to validate and connect all the filters following a pipeline of streams that compose a filter graph artifact template. The connection between filters is represented by a vector of connections, Connections, in which each link is an ordered pair consisting of the index into the vector of filter types and the respective filter type.
4.20 Listing 22 Ordered pair vector specifying connections between CRTSPSource, CJPEGDec and CXVRenderer filters

$1 / /$ filter types vector

2 typedef mpl::vector $<$ CRTSPSource, CJPEGDec, CXVRenderer $>$ Types;

3

$4 .$.

5 // pair that represents the link between CRTSPSource and CJPEGDec filter

6 typedef mpl::pair $<$ mpl::int_<0 $>$, mpl::int_<1 $>>$ c1;

7 // pair that represents the link between CJPEGDec and CXVRenderer filter

8 typedef mpl::pair $<$ mpl::int_<1>, mpl::int_<2>>c2; 9

10 // filter connections vector

11 typedef mpl::vector $<\mathrm{c} 1, \mathrm{c} 2>$ Connections;

Listings 23, 24 and 25 specify how the links described by the Connections vector are applied during the filter graph construction by an auxiliary function template, ConnectFilters (see Listing 24), which receives a Connection vector as input parameter. At the instantiation time, a specialized or concrete CFilterGraph class should implements the ConnectFilters function template which uses the inline ConnectFilters of the FilterGraphManager structure given by Listing 25 .

\subsection{Listing 23 Building a filter graph stream by connecting a set of filters \\ 1 // filter graph variable \\ 2 CFilterGraph $<$ Types, mpl::size $<$ Types $>:$ value $>$ fg; \\ 3 // connect filters \\ 4 fg.ConnectFilters $<$ Connections $>($ );}

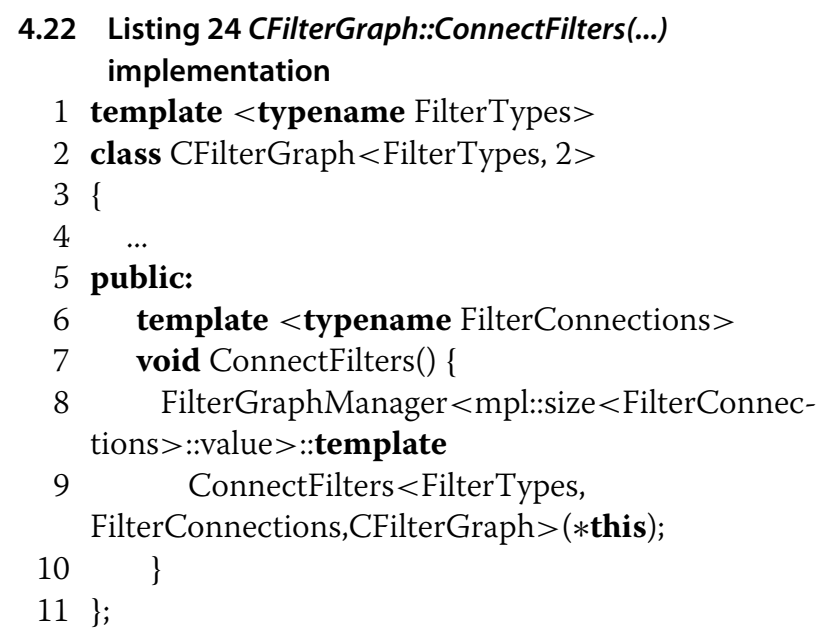

4.23 Listing 25 Template struct FilterGraphManager implemented as a filter graph manager

1 template $<$ int $N>$ struct FilterGraphManager; 
2

3 template $<$ int $\mathrm{N}>$

4 struct FilterGraphManager \{

5 template $<$ typename Types, typename

Connections, typename FilterGraph $>$

6 inline static void ConnectFilters(FilterGraph\& fg) \{

7 // TODO: add code to connect filters recursivelly

$8 \quad\}$

9

10

11

template $<$ typename FilterGraph $>$ inline static void StartFilters(FilterGraph\& fg) \{ // TODO: add code to start filters recursivelly \}

template $<$ typename FilterGraph $>$ inline static void StopFilters(FilterGraph\& fg) \{ // TODO: add code to stop filters recursivelly \}

template $<$ typename FilterGraph $>$ inline static void PauseFilters(FilterGraph\& fg) \{ // TODO: add code to pause filters recursivelly \} \}

template $<>$

struct FilterGraphManager $<0>\{$

template $<$ typename Types, typename

Connections, typename FilterGraph $>$

29 fg) \{\}

inline static void ConnectFilters(FilterGraph\& 30 39 ;

template $<$ typename FilterGraph $>$ inline static void StartFilters(FilterGraph\& fg) \{\}

template $<$ typename FilterGraph $>$ inline static void StopFilters(FilterGraph\& fg) \{\}

template $<$ typename FilterGraph $>$

inline static void PauseFilters(FilterGraph\& fg) \{\}

The FilterGraphManager template structure in Listing 25 implements the management of certain filter graph operations, such as, filter connections and start, stop, and pause of the filter streams execution. The inline functions ConnectFilter, StartFilters, StopFilters and PauseFilters connects two filters of a given filter graph, starts the filters execution flow, stops the filters execution flow and pauses the filters execution flow, respectively.

Since the management operations are applied recursively with the number of operations given by $N$, the FilterGraphManager structure is implemented by a generic template (line 05 to 28 ) and a specialized one with input parameter zero (line 31 to 45 ) to indicate the termination condition. When $N$ is zero, all inline functions defined inside the specialized template will present an empty body and the $\mathrm{C}++$ compiler patches no code at all.

The ConnectFilter inline function of FilterGraphManager structure accepts three template parameters (i.e., Types, Connections, FilterGraph) and a filter graph reference as input parameter to connect the filters composing a filter graph as shown by its implementation in Listing 26.

4.24 Listing 26 Inline ConnectFilters function code 1 template $<$ int $\mathrm{N}>$ struct FilterGraphManager;

2

3 template $<$ int $\mathrm{N}>$

4 struct FilterGraphManager \{

5 template $<$ typename Types, typename Connections, typename FilterGraph $>$

6 fg) \{ inline static void ConnectFilters(FilterGraph\&

7

8$$
9
$$

10

11

mpl::at $<$ Connections,mpl::long_<N-1> > :type elem;

12

$$
13 \begin{gathered}
\text { mpl::first }<\text { elem }>\text { ::type }>\text { ::type } \\
\text { out_filter_type; }
\end{gathered}
$$

14 typedef typename mpl::at< Types, mpl::second $<$ elem $>$ ::type $>:$ :type

15 in_filter_type;

16

17 out_filter_type,

18 in_filter_type $>$;

20 // connect filter first $<$ elem $>$ to second $<$ elem $>$ ; elem $<A, B>$

21 fg.GetFilter(mpl::first $<$ elem $>$ ::type()).

Connect(\&fg.GetFilter(mpl::second <elem>::type()), 22 ::type()).Process); fg.GetFilter(mpl::second < elem> 


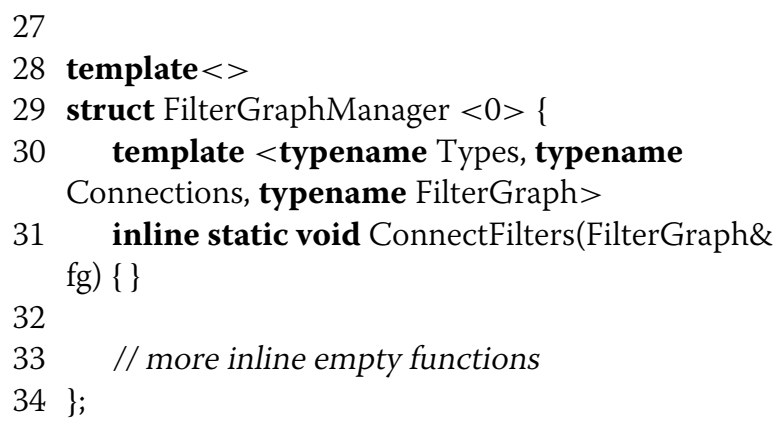

In each iteration is called first the same function recursively with value $N-1$ (line 11), then a test is run to validate the connection between two filters (lines 20 to 21) and finally the connection is made (line 24 to 25 ). As an example, consider the filter graph, the code to create it and the recursive iteration inside ConnectFilters method for the filter graph $f g$ as shown in Figures 11, 12 and Listing 27, respectively.

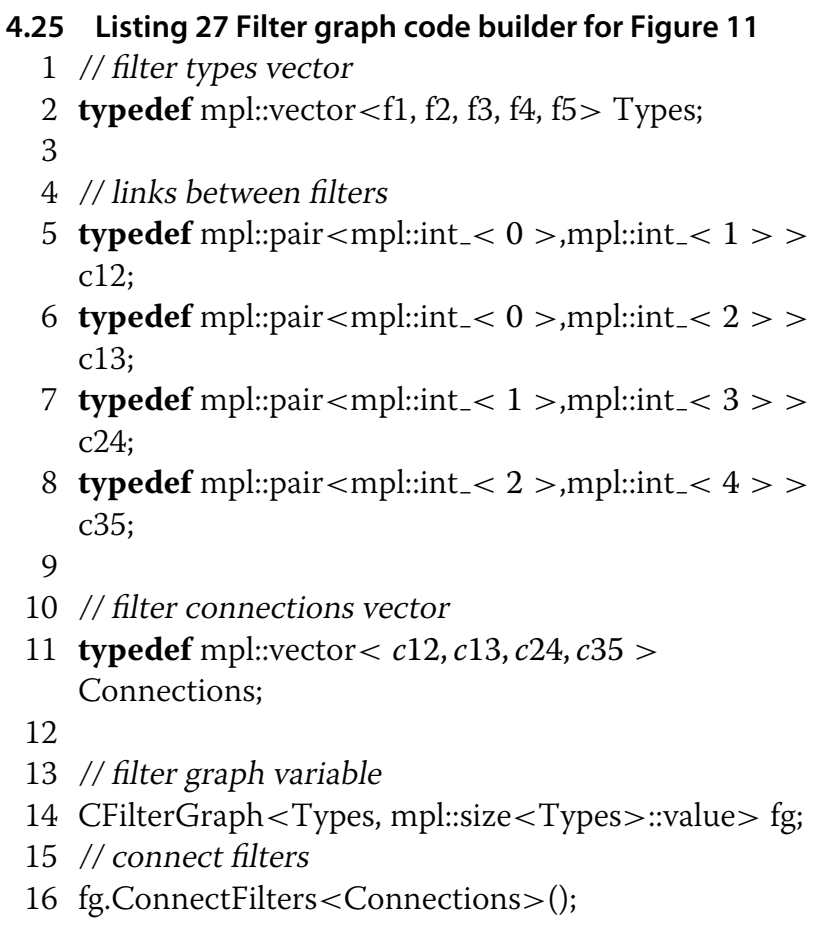

8 typedef mpl::pair $<$ mpl::int_< $2>$, mpl::int_< $4>>$ c35;

$10 / /$ filter connections vector

11 typedef mpl::vector $<c 12, c 13, c 24, c 35>$ Connections;

12

13 // filter graph variable

14. CFilterGraph <Types, mpl::size $<$ Types $>::$ value $>$ fg;

15 // connect filters

16 fg.ConnectFilters $<$ Connections $>()$;

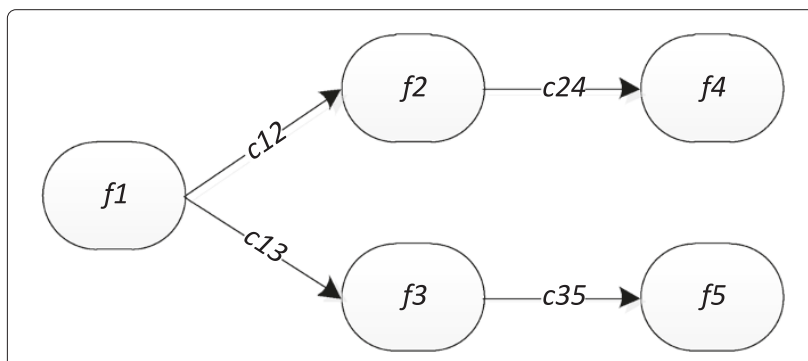

Figure 11 Filter graph with five filters.
The StartFilters, StopFilters or PauseFilters inline functions template of the FilterGraphManager structure given by Listings 28, Listing 29 Inline StopFilters function code and 30, performs only two operations. The first one will recursively call the Start, Stop or Pause methods of filters at indexes lower than $N-1$ and greater than zero while the second one executes the Start, Stop or Pause methods of the filter at index $N-1$.

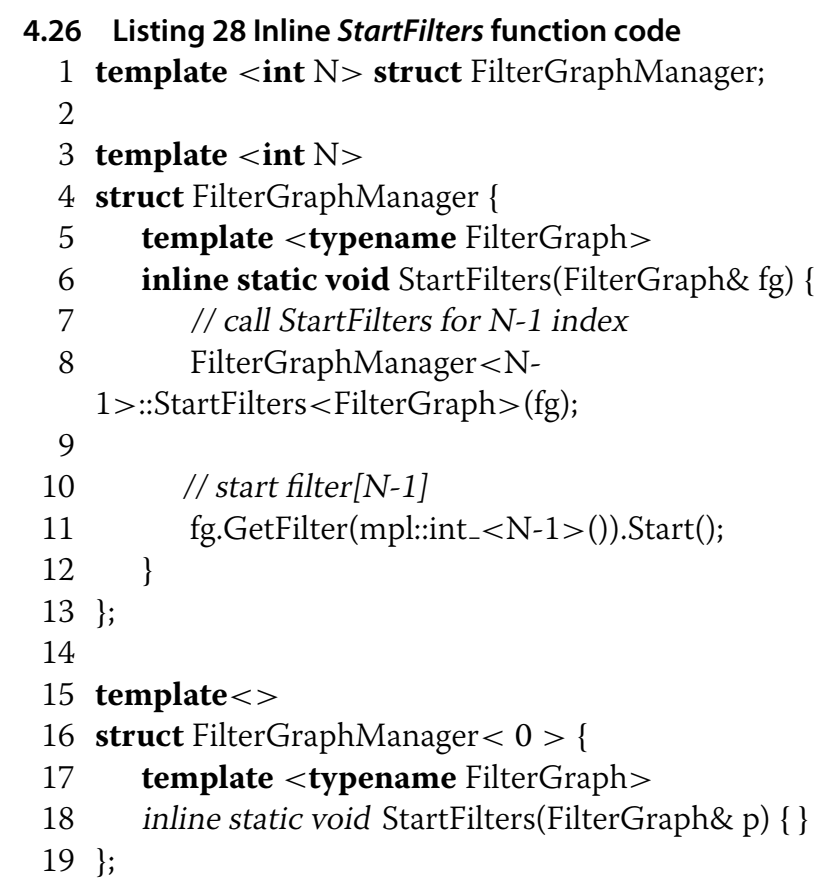

\subsection{Listing 29 Inline StopFilters function code} 1 template $<$ int $\mathrm{N}>$

2 struct FilterGraphManager \{ template <typename FilterGraph $>$ inline static void StopFilters(FilterGraph\& fg) \{ // call stop_filters for $N-1$ index FilterGraphManager $<\mathrm{N}$ $1>$ ::StopFilters $<$ FilterGraph $>(\mathrm{fg})$; 7 $8 \quad / /$ stop filter $[N-1]$ 9 fg.GetFilter(mpl::int_<N-1 $>())$.Stop(); 10 11 \}; 12 13 template $<>$ 14 struct FilterGraphManager $<0>\{$ 15 template $<$ typename FilterGraph $>$ 16 inline static void StopFilters(FilterGraph\& p) \{\} 17 ;

\subsection{Listing 30 Inline PauseFilters function code} 1 template <int $\mathrm{N}>$ struct FilterGraphManager; 2 3 template $<$ int $\mathrm{N}>$ 
fg.ConnectFilters $<$ Connections $>($ );

FilterGraphManager $<4>$ ::template

ConnectFilters $<$ Types, Connections, CFilterGraph $>(\& f g)$;

FilterGraphManager $<3>:$ :template

ConnectFilters $<$ Types, Connections, CFilterGraph $>(\& f g)$;

FilterGraphManager $<2>:$ :template

ConnectFilters $<$ Types, Connections, CFilterGraph $>(\& f g)$;

FilterGraphManager $<1>$ :: template

ConnectFilters $<$ Types, Connections, CFilterGraph $>(\& f g)$;

FilterGraphManager $<0>$ ::template

ConnectFilters $<$ Types, Connections, CFilterGraph $>(\& f g)$;

fg.at $($ mpl:::int_<0>).Connect(\&fg.at(mpl::int_<1>), fg.at(mpl::int_<1>).process);

fg.at(mpl::int_<0>).Connect(\&fg.at(mpl::int_<2>), fg.at(mpl::int_<2>).process);

fg.at(mpl::int_<1>).Connect(\&fg.at(mpl::int_<3>), fg.at(mpl::int_<3>).process);

fg.at(mpl::int_<2>).Connect(\&fg.at(mpl::int_<4>), fg.at(mpl::int_<4>).process);

f1. connect(\&f2, f2.process);

f1.connect(\&f3, f3. process);

f2. connect $(\& \mathrm{f} 4$, f4. process);

f3.connect $(\& f 5$, f5. process);

Figure 12 ConnectFilters function iterations.

4 struct FilterGraphManager \{

5 template $<$ typename FilterGraph $>$

6 inline static void PauseFilters(FilterGraph\& fg) \{

7 // call pause_filters for $N-1$ index

8 FilterGraphManager $<$ N-

$1>::$ PauseFilters $<$ FilterGraph $>(\mathrm{fg}) ;$

9

10 // pause filter[N-1]

11 fg.GetFilter(mpl::int_<N-1>()).Pause();

$12\}$

$13\}$;

14

15 template $<>$

16 struct FilterGraphManager $<0>\{$

template $<$ typename FilterGraph $>$

inline static void PauseFilters(FilterGraph\& p) \{\}

\subsubsection{The intelligent connect artifact template}

The AllowConnection artifact template, shown in Listing 31 , was implemented to validate the connection between two filters based on the following conditions: (1) the input and output filters should present at least one output and input pin respectively, (2) the two pins through which the connection is made should support the same type of data stream, and (3) the minimum stream requirement dictated by at least one source and output filters with compatible media type. It receives as parameters the data types of the two filters, i.e., one audio/video format vector supported at the input of InputFilter and another one at the output of OutputFilter.

\subsection{Listing 31 AllowConnection template class code \\ 1 template $<$ typename OutputFilter, typename InputFilter $>$ \\ 2 class AllowConnection \{ \\ 3 typedef typename OutputFilter::output_types output_types; \\ 4 typedef typename InputFilter::input_types input_types; \\ 5 \\ 6 public:}




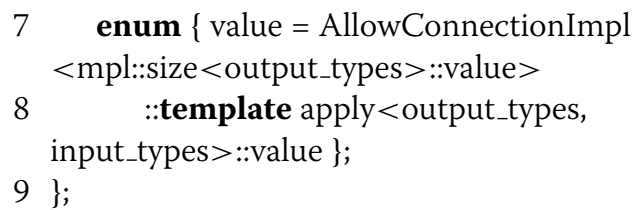

Listing 32 partially presents the implementation of AllowConnectionImpl template structure that registers the result of connecting two filters into the enumeration variable value. This structure template is implemented by a specialized and a generic template, both receiving as template input parameter the number of elements of the output_types vector related to the OutputFilter. Also, both templates define a metafunction, apply, that takes the audio/video vectors supported by each filters as input parameters. For each element in the output_types vector it uses a boost MPL library metafunction named contains to check if the same element exists in the input_type vector. Any denied connection is verified and notified with an error message by the CheckErrorOnConnection template structure shown in Listing 33.

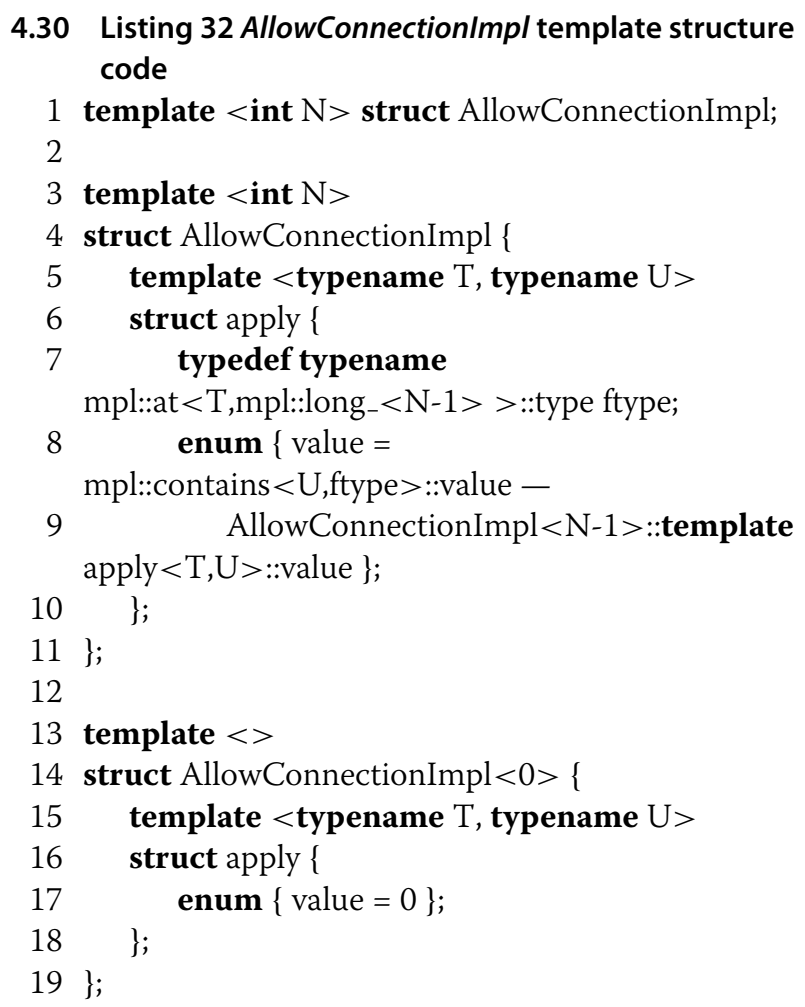

\subsection{Listing 33 CheckErrorOnConnection template} structure code

1 // forward declaration

2 template $<$ bool $\mathrm{C}$, typename $\mathrm{T}$, typename $\mathrm{U}>$ struct CheckErrorOnConnection;

3

4 // generic implementation
5 template $<$ bool $\mathrm{C}$, typename $\mathrm{T}$, typename $\mathrm{U}>$

6 struct CheckErrorOnConnection

7\{

8 static_assert(C, "cannot connect the two filters."); 9 ;

10

11 // template specialization for filters v4l2 and xvideo

12 template $<$ bool $\mathrm{C}>$

13 struct CheckErrorOnConnection $<$ C,CV4L2Source, CXVRenderer>

14\{

15 static_assert(C, "cannot connect v4l2 filter to xvideo filter.");

16 \};

17

18 // specializations for the other filters combinations $19 \ldots$

A denied connection occurrence is indicated by one of the three template parameters received by CheckErrorOnConnection template structure, i.e., the first one that has a boolean type. If denied, the value of $C$ will be false as well as the input parameter of static_assert function and so, forcing a compilation error with our predefined message. For a more complete notification of a denied connection, specialized templates are defined, combining all participating filters.

The domain model will be concluded with the built-in intelligent connect feature of the filter graph composition and validation that is in charge of the following three tasks: (1) trying combinations of intermediate transform filters to satisfy the required matching between a pair of output and source pins, (2) specializing some partially configured filter that failed to be fully mapped to a model, and (3) avoiding code bloat by exploring any possible cross-silo synergy among similar filters. Possible code bloat may happen with a video surveillance system consisting of more than one filter graph using the same filter but with different configurations. In such case, several different classes will be instantiated, one for each configuration as illustrated in Figure 13.

To tackle the code bloat phenomenon, intelligent connect initially analyzes the type of filters and their settings for each pipelines and create a list of setting for any repeated filter among different pipelines but with different settings. Based on the collected settings, a unique class will be created, instantiated and shared among pipelines, as shown in Figure 14.

To conclude the domain engineering stage of the video surveillance systems development environment, a XSLT code transformer, shown in Listing 34, was implemented to generate a Config.h file from the configuration. This file will be used later by the offline partial evaluator $\mathrm{C}++$ 

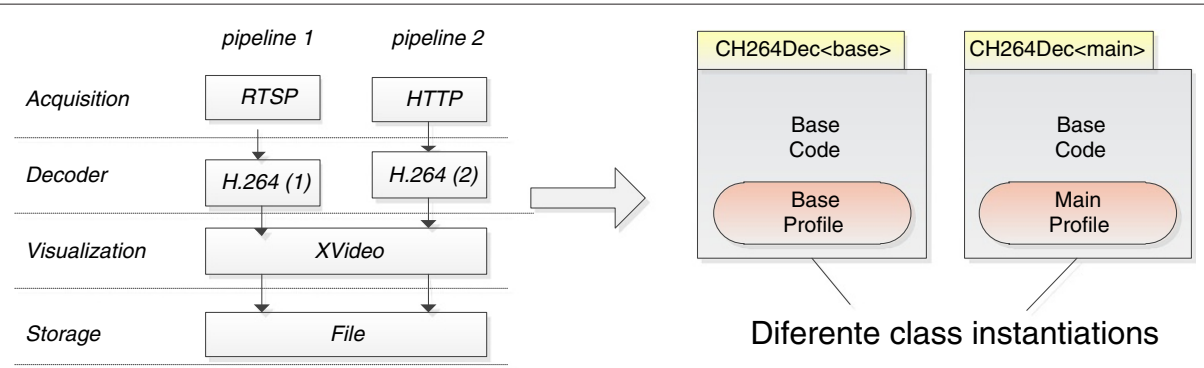

Figure 13 Bloat due to H.264 filter duplication with base and main profiles.

code generator that is in charge of the filter graph artifact template instantiation.

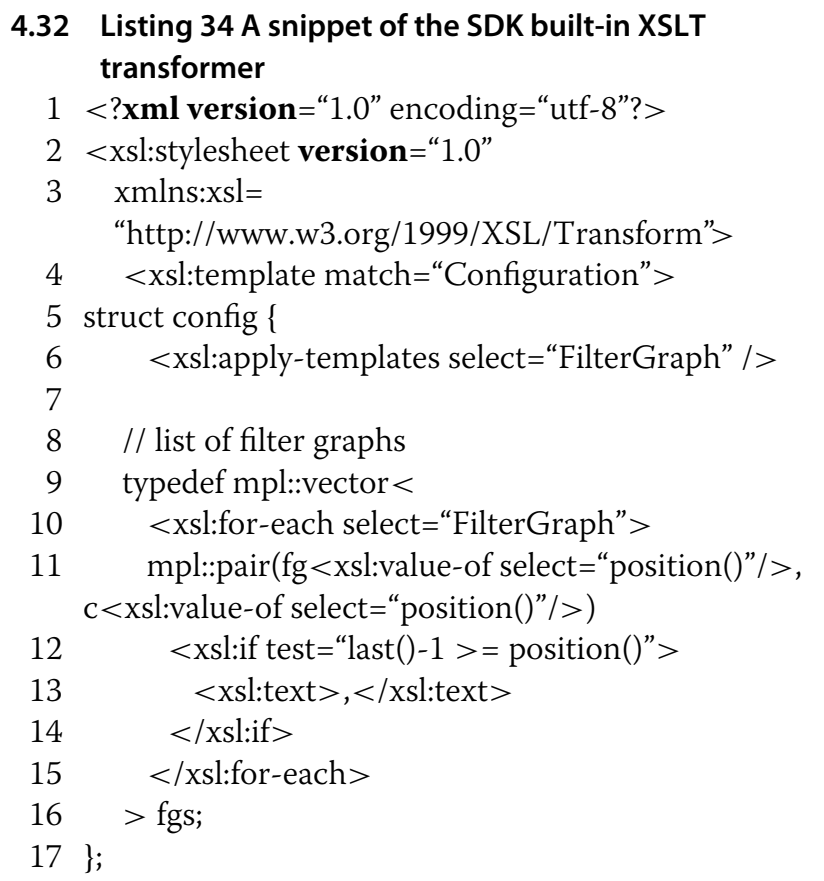

$$
\begin{array}{ll}
18 & </ \text { xsl:template }> \\
19 & \ldots \\
20 & </ \text { xsl:stylesheet }>
\end{array}
$$

\section{Video surveillance application engineering}

The passage from development for reuse to the development with reuse of video surveillance systems requires several steps surrounded by two main activities as shown by Figure 15: (1) establishing the communication channel between the stakeholders (IVV Automation customers) and system analysts and programmers to build a model family instance (i.e., a feature configuration and model templates), and then (2) generating the final code from the configured model family. At the end of a video surveillance system configuration process and before starting the code generation process, several concrete filters and filter graph will be encoded in XML and validated against the generic ones, and a mapping will be intensive and automatically established between features of the feature model and the model elements of the domain model.

Our MDD approach combines feature models and domain models on the artifact template class level encoded using $\mathrm{C}++$ template metaprogramming. The mapping is automatically done at three level of granularity, i.e., at artifact template filter graph, at artifact template

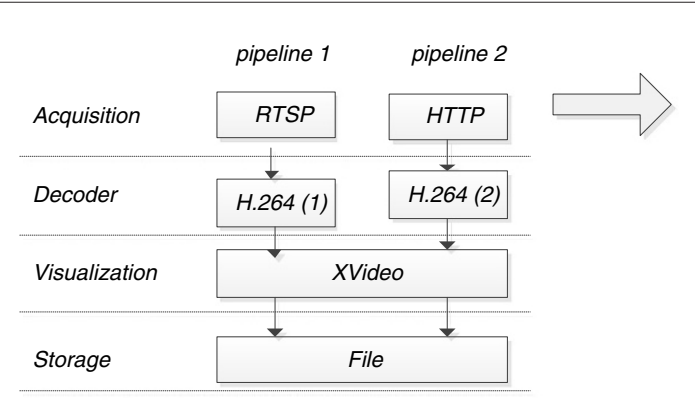

PréProcessador de Instanciações: $\mathrm{CH} 264 \mathrm{Dec}<$ base $>+\mathrm{CH} 264 \mathrm{Dec}<$ main $>$

CH264Dec <vector<base, main $>>$
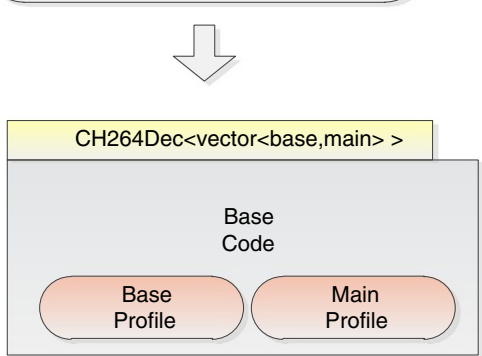

Figure 14 Optimized Code after intelligent connect intervention. 


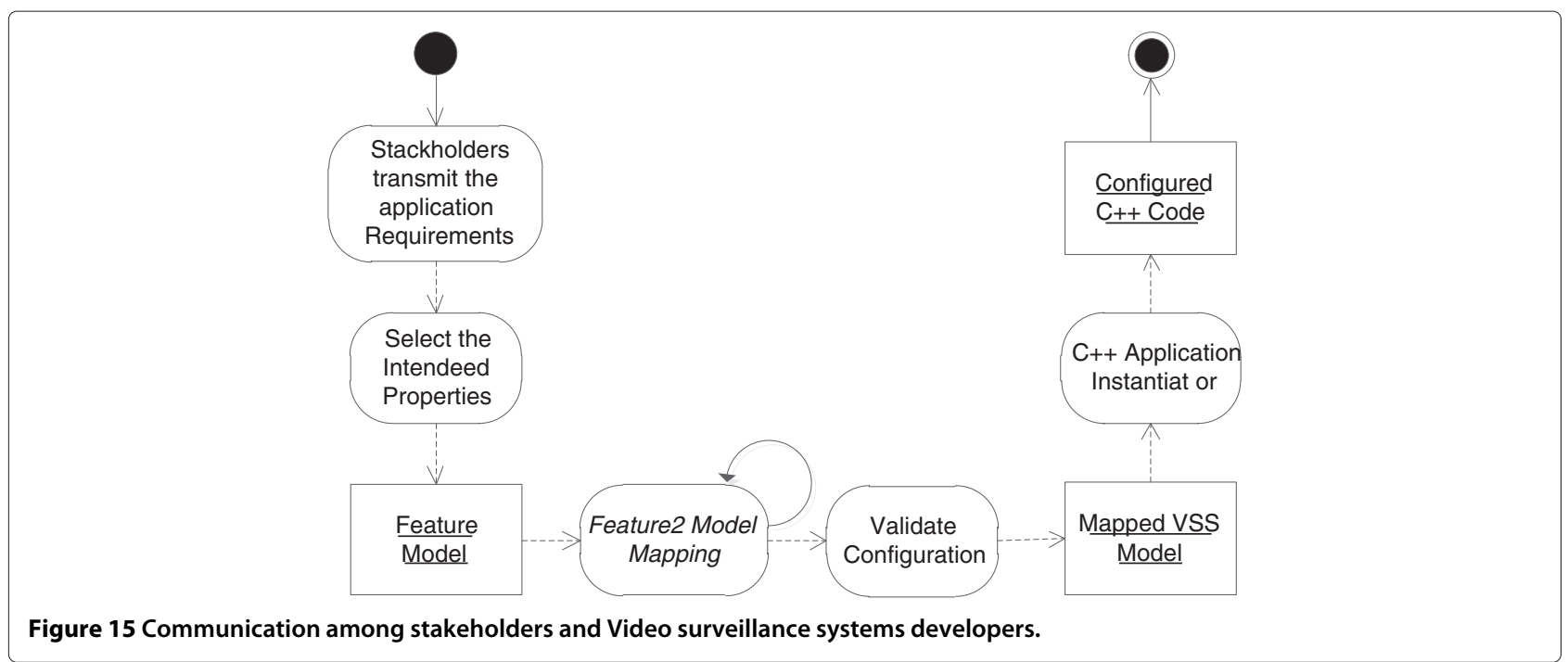

filter and internally to the artifact template filter at several variability points, such as attributes and methods when in the sync mode is selected or semi-automatically otherwise. Selected features of a filter will be successive collected till the moment that the filter can be evaluated by a meta-expression to a particular artifact template filter and the mapping realized. Any other selected features will be mapped to internal variability points or attributes of the previously mapped artifact template filter by metaexpressions or presence conditions, respectively, indicating whether they should be patched or removed from the specialized template instance. Mapping at template filter graph level will be triggered by a meta-expression when at list two filters are linked in a stream. Missing or unmapped feature in the domain model forces the extension of the domain model and so, be carefully analyzed. By now, the online partial evaluator based on $\mathrm{C}++$ template metaprogramming solved it successfully, after refactoring some filter legacy code. Figure 16 shows a simple example of our Feature2ModelMapping activity.

Listings 35, 36 and 37 shows the second step of a two step video surveillance system generator, as the first one is a SDK built-in functionality. First, the XSLT transformer is fed with a video surveillance system configuration to generate the Config.h and then calling an offline partial evaluator based on $\mathrm{C}++$ template metaprogramming (i.e., the CApplicationInstantiator), some of the previously artifact templates produced during domain engineering will
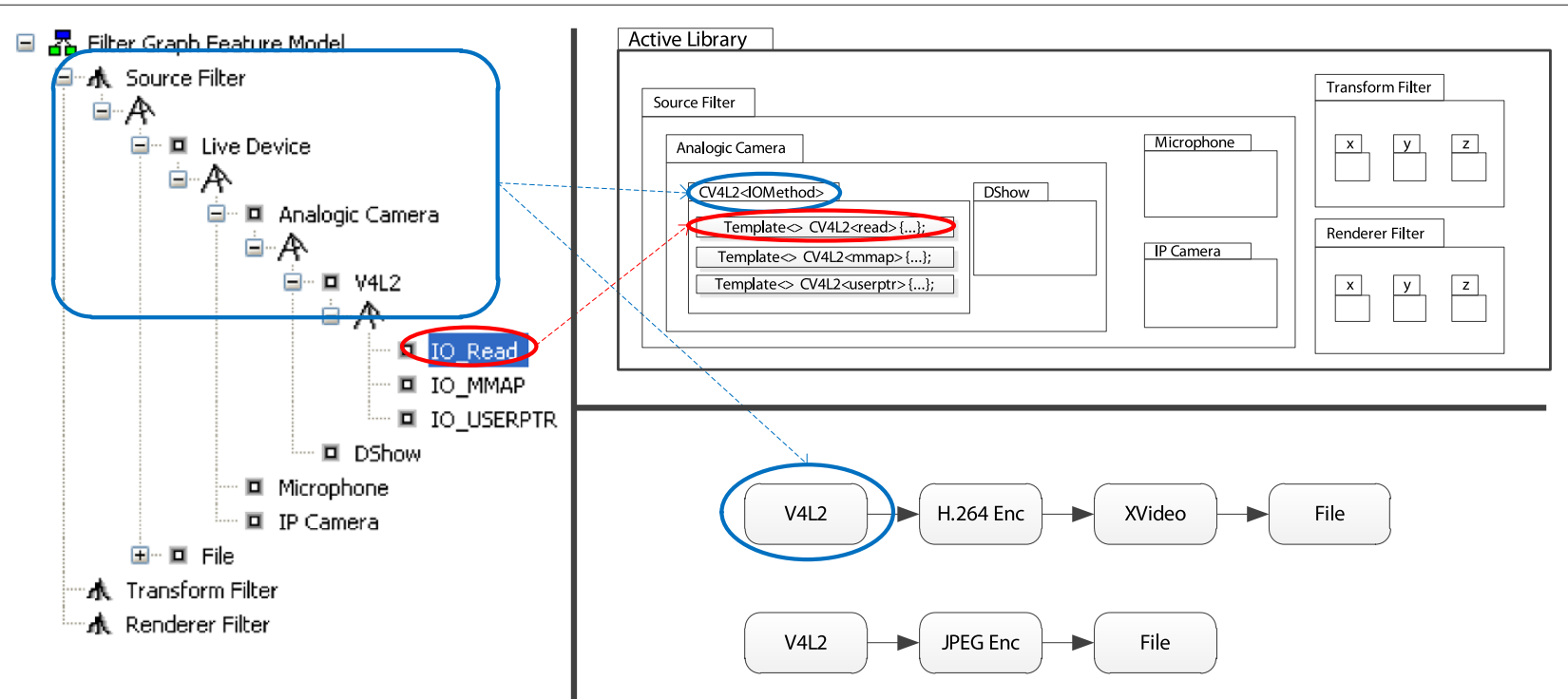

Figure 16 Exemplifying the SDK built-in feature to model mapping. 
be customized into specialized application-specific components and linked together to construct the final video surveillance system that addresses the need of specific customers. Basically, the offline partial evaluator carries out the instantiation process at several levels of granularity by translating the video surveillance system configuration into an ordered sequence of finer instantiation operations which when executed will instantiate the new video surveillance system.

\subsection{Listing 35 Offline Partial Evaluator: the} CApplicationInstantiator class

1 template $<$ typename Config $>$

2 class CApplicationInstantiator

3 : CApplicationInstantiatorImpl $<$ IntelligentConnect $<$ Config::fgs $>::$ value, mpl::size $<$ IntelligentConnect $<$ Config::fgs $>$ ::value $>>\{\}$
5.2 Listing 36 A snippet of the Offline Partial Evaluator: the CApplicationInstantiatorlmpl class

1 template < typename FilterTypes, int $\mathrm{N}>$ class CApplicationInstantiatorImpl;

2

3 template <typename Vector $>$

4. class CApplicationInstantiatorImpl $<$ Vector, $1>\{$

5 typedef typename mpl::at $<$ Vector, mpl::long_<0>>::type item0;

6 typedef typename mpl::first $<$ item $0>:$ :type Filters0;

7 typedef typename mpl::second <item0>::type Connections0;

8

9 CFilterGraph $<$ Filters0, mpl::size $<$ Filters0 $>>$ fg0;

10 public:

11 void Initialize() \{

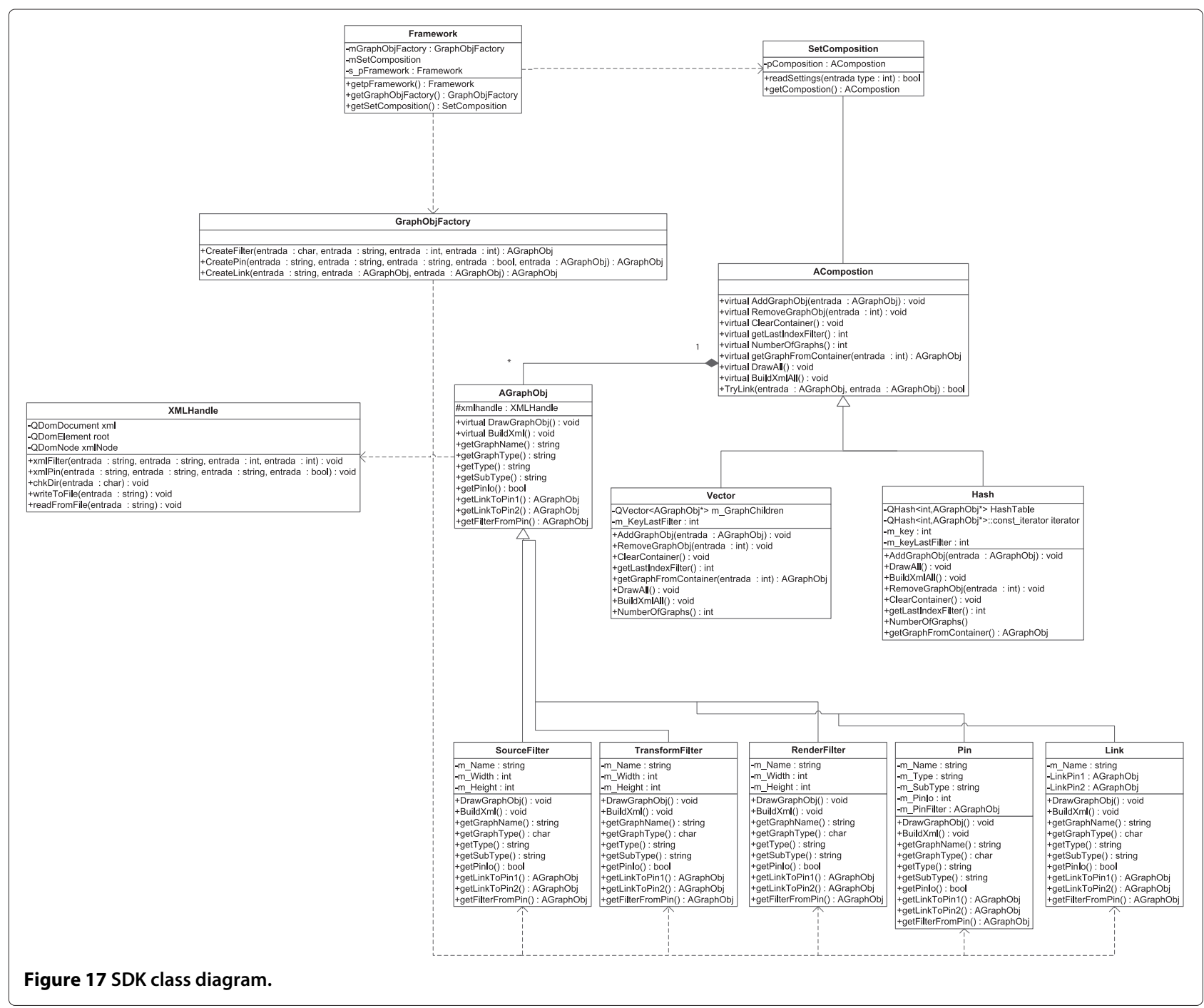




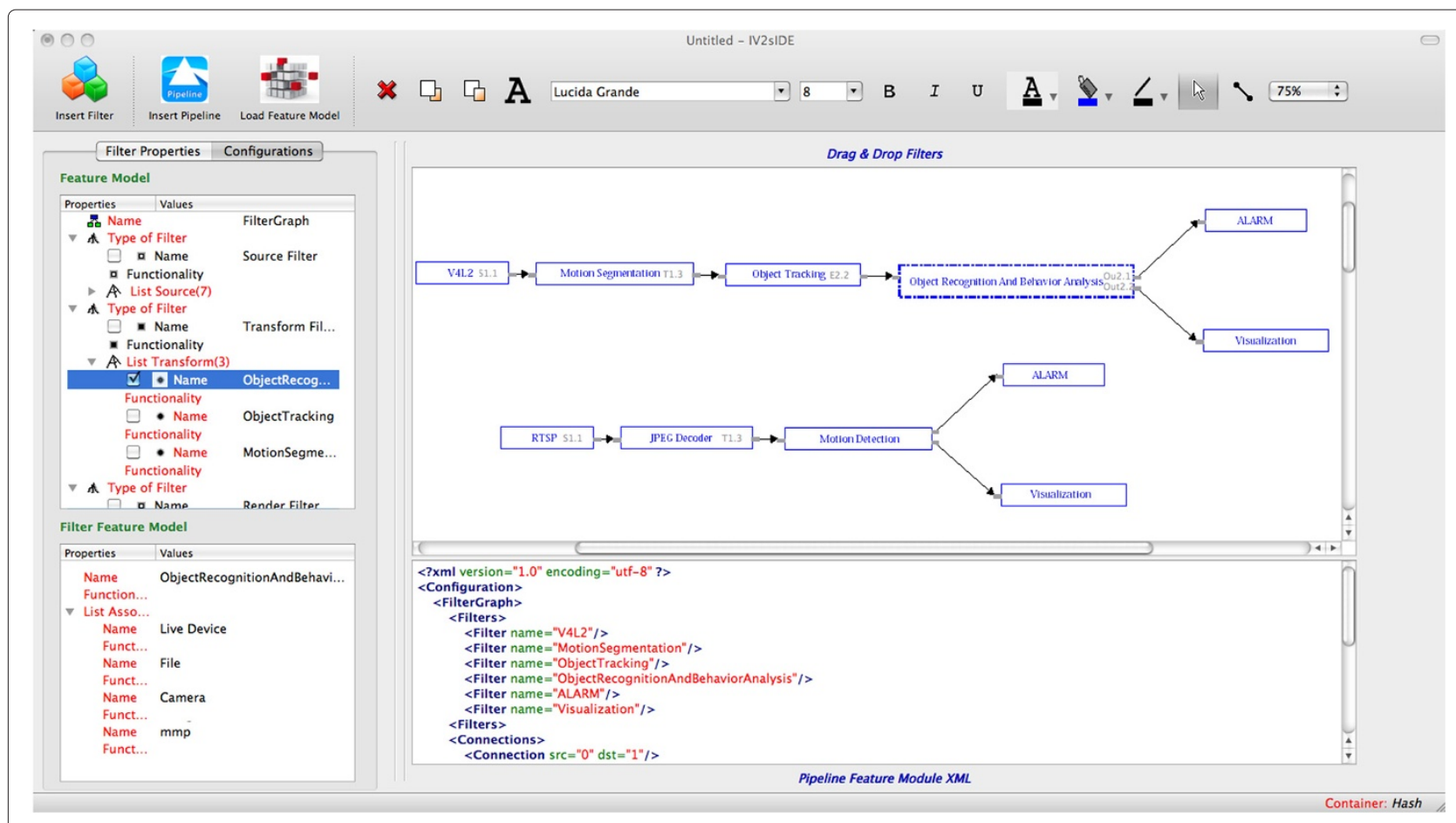

Figure 18 Screenshot of the SDK exemplifying the design of two filter graphs.

16 template <typename Vector $>$

17 class CApplicationInstantiatorImpl $<$ Vector, $2>\{$

18 typedef typename mpl::at $<$ Vector, mpl::long_ $<0>>$ ::type item0;

19 typedef typename mpl::at $<$ Vector, mpl::long_<1>>::type item 1 ;

20 typedef typename $\mathrm{mpl}:$ :first $<$ item $0>$ ::type Filters0;

21 typedef typename mpl::second <item0>::type Connections0;

22 typedef typename mpl::first<item $1>$ ::type Filters1;

23 typedef typename mpl::second <item $1>$ ::type 24

\section{Connections1;}

25 CFilterGraph $<$ Filters0, mpl::size $<$ Filters0 $>>$ fg0;

26 CFilterGraph $<$ Filters1, mpl::size $<$ Filters1 $>>$ fg1;

27 public:

28 void Initialize() \{

29 fg0.ConnectFilters $<$ Connections $0>($ )

30 fg1.ConnectFilters $<$ Connections1 $>$ ();

$31\}$

$32\}$

33 ...

\subsection{Listing 37 Offline Partial Evaluator: $\mathrm{C}++$ final code} generation

1 \#include < config.h $>$

2

3 int main $($ int $\operatorname{argc}$, char $* \operatorname{argv}[])$

4\{

5 CApplicationInstantiator app $<$ Config $>$;

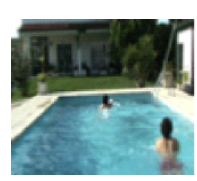

Raw video

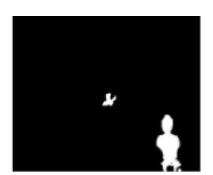

Segmentation

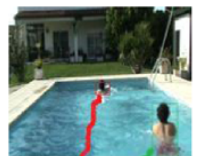

Tracking

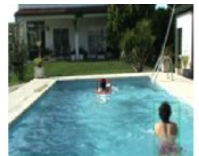

Identification

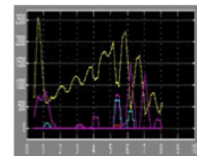

Pattern Recognition

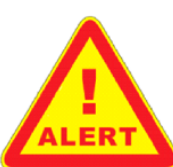

Alert and Visualization

Figure 19 Activities sequence of the early drowning detection at domestic swimming pools. 


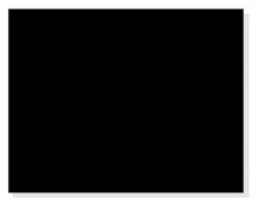

Compressed video

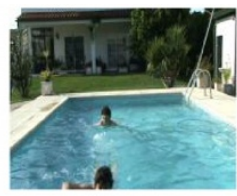

Raw video

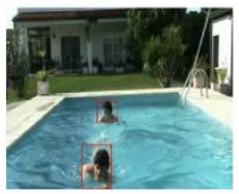

Motion Detection

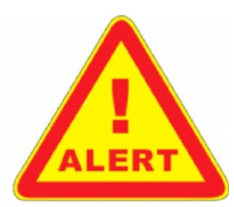

Alert

and Visualization

Figure 20 Motion detection at domestic swimming pools.

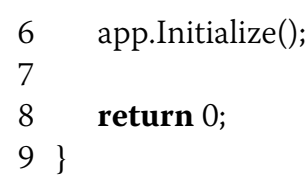

\section{Video surveillance product line SDK}

A Qt-based video surveillance system SDK that allows graphical instantiation of a feature model configuration similar to DirectShow graphEdit was designed using several design patterns, such as singleton, strategy, factory and composite, as shown in Figure 17. After a configuration instantiation all the previously described steps will be executed till the generation of final $\mathrm{C}++$ configured code for the new video surveillance system. Figure 17 presents a screenshot of the design of an early drowning detection at domestic swimming pools consisting of two pipelines: the first one with six filters:

(i) An analog input filter, V4L2, for image capture;

(ii) Three transform filters for motion segmentation, object tracking, and object recognition and analysis behavior;

(iii) Two output filter for alarm and visualization.

The second pipeline has the following five filters:

(i) A digital input filter, RTSP, that captures image frames from an IP camera using RTSP/RTP for transmission;

(ii) Two transformation filters for JPEG decoding and motion detection;

(iii) Two output filter for alarm and visualization.

Figure 18 illustrates two of several possible functionality scenarios supported by the generated system of Figures 19 and 20.

\section{Methods}

As previously mentioned, the video surveillance ecosystem is divided in two stages: the implementation domain, developed envisioning the system evolution, followed by the application engineering stage.

During the application engineering stage, a specific pipeline was created in order to meet the client imposed application requirements. In the pipeline creation process, several alternatives were explored, both at low levels (e.g., platform selection) and at high levels (e.g., filter selection according to context aware strategies.)

In this specific case, to make the comparison study between the two implementations, a video surveillance system based on DirectShow using $\mathrm{C}++$, with virtual functions and inheritance, and a video surveillance system using the proposed framework were used on two kinds of machines. 1) First, a general purpose PC was used, an Intel Core 2 Duo CPU T9550 at $2.66 \mathrm{GHz}$ with $2,83 \mathrm{~GB}$ of RAM with Ubuntu 10.04 and secondly an embedded system based on a BeagleBoard-xM with an ARM CortexA $8 \mathrm{MHz}$ at $1 \mathrm{GHz}$ and extra memory with $512 \mathrm{MB}$ of low-power DDR RAM with Angstrom Embedded Linux.

For the two configurations, a pipeline composed by an RTSP source filter, a JPEG decoder transform filter and a File output filter was created. The pipeline was compiled with the GNU $\mathrm{C}++$ compiler $(\mathrm{g}++)$ with the $-\mathrm{O} 3$ optimization option.

\section{Conclusion and future work}

To meet the embedded systems constraints and tackle the growing complexity of new video surveillance systems and time-to-market pressure, we abandoned our previous product-centric perspective and shifted to the multitude of products. In doing so, we promote the integration of MDD, SPL and generative technologies to create software for a portfolio of video surveillance systems in a pay-asyou-go fashion rather than an ex-nihilo development. The synergistic convergence of those technologies improved our ability to meet product quality demands while reducing the development effort and expanding the scale and scope of our video surveillance systems portfolio. One of the unique features of the proposed video surveillance systems design environment compared to existing ones, is its simplicity and higher integration level of mainstream tools and technologies such as, XML, XSLT, C++ template metaprogramming, generative programming, $\mathrm{MDD}$, and SPL. Acher's et al. proposal didn't discuss the code generation process, i.e., it seems to us like an under development work. The proposed system supports the easy integration of external libraries that implement wellknown standards and functionalities, e.g., ONVIF and 
PSIA, developed as a filter that is integrated on the video surveillance system. The system was tested on a Beagle Board. The core processor of this embedded platform is the Texas OMAP that includes a DSP capable of decompressing HD frames in H.264 format, for which an encoding/decoding filter was developed. When the hardware system doesn't support SIMD instructions for video processing (MMX, SSE2, etc) or is not equipped with a DSP, then the video surveillance system performance will be degraded.

However, this project is still under development and next steps will be focused on: (1) automating the simulation and verification of the generated code, (2) automating the test cases generation and testing of the generated filters and video surveillance systems, (3) the integration of external modules, developed in other programming languages such as Python or Java (i.e., JNI-aware $\mathrm{C}++$ wrapper), that implement video surveillance functionalities, and (4) study of the variability management of functionality related to playback application, e.g., playback, jump to absolute timestamp and find nearest I-Frame.

\section{Endnote}

${ }^{a}$ http://www.ivv-aut.com/.

\section{Competing interests}

The authors declare that they have no competing interests. This work was funded by the Science and Technology Foundation (FCT) and developed in cooperation with IW Automation, but none pose any objection to the publication of results.

\section{Acknowledgements}

This work was funded through the Competitive Factors Operational Program COMPETE and through national funds though the Science and Technology Foundation - FCT, within the project: FCOMP-01-0124-FEDER-022674. This work was developed in cooperation with IV Automation; all support and means provided by the company is acknowledged.

\section{Author details}

'Department of Industrial Electronics, Centro Algoritmi, University of Minho, Braga, Portugal. ${ }^{2}$ School of Engineering and Technology, Asian Institute of Technology, Khlong Luang, Thailand.

Received: 23 January 2012 Accepted: 5 June 2012

Published: 31 July 2012

\section{References}

1. L Marcenaro, L Marchesotti, C Regazzoni, in Proceedings of the Fifth International Conference on Information Fusion. vol. 2. A multi-resolution outdoor dual camera system for robust video-event metadata extraction, (Annapolis, MD, Washington DC Area, USA, 2002), pp. 1184-1189

2. M Acher, $P$ Lahire, S Moisan, JP Rigault, in Modeling in Software Engineering, 2009. ICSE Workshop on MISE '09. Tackling high variability in video surveillance systems through a model transformation approach, (Vancouver, Canada, 2009), pp. 44-49

3. I Ahmad, Z He, M Liao, F Pereira, MT Sun, Special issue on video surveillance, IEEE Trans. Circ. Syst. Video Technol. 18(8), 1001-1005 (2008)

4. Y Hongyu, X Lixia, X Feng, in IEEE International Conference on Industrial Informatics. Research on cluster remote video surveillance system, (Singapore, 2006), pp. 1171-1174

5. MD Pesce, Programming Microsoft DirectShow for Digital Video and Television. (Microsoft Press, Redmond, Washington, 2003)
6. PL Plauger, Embedded C++: An Overview. Embedded Systems Programming, (1997). [http://www.dsi.fceia.unr.edu.ar/downloads/informatica/info_ll/ $\mathrm{c}++$ /ec++.pdf]

7. S Meyers, Effective $\mathrm{C}++$ in an embedded environment. [http://www. aristeia.com/c++-in-embedded.html]

8. D Saks, in Embedded Systems Conference. Reducing run-time overhead in C++ programs, (San Francisco, USA, p. 2002

9. 9. Technical Report on C++ Performance. [www2.research.att.com/bs/ performanceTR.pdf]

10. I McRitchie, TJ Brown, ITA Spence, Managing component variability within embedded software product lines via transformational code generation, Softw. Product Family Eng. 3014/2004, 98-110 (2004)

11. A Kleppe, J Warmer, W Bast, MDA Explained: The Model Driven Architecture: Practice and Promise. Addison-Wesley Professional, Boston, 2003)

12. K Czarnecki, U Eisenecker, Generative Programming: Methods, Tools, and Applications. (Addison-Wesley Professional, Boston, 2003)

13. R Anisko, Towards automatic partial evaluation for the $\mathrm{C}++$ language, in CSI Seminar (2002)

14. D Lohmann, W Hofer, W Schroder-Preikschat, O Spinczyk, in AOSD'1 Proceedings of the tenth international conference on Aspect-oriented software development. Aspect-aware operating system development, (Porto de Galinhas, Pernambuco, Brasil, 2011), pp. 69-80

15. V Sarinho, A Apolinario, in SBGAMES '09 Proceedings of the 2009 VIII Brazilian Symposium on Games and Digital Entertainment. A generative programming approach for game development, (Rio de Janeiro, Brazil 2009), pp. 83-92

16. T Buchmann, A Dotor, in Pieter van Gorp, Hrsg., Proceedings of the 7th International Fujaba Days. Mapping features to domain models in Fujaba, (Seiten 20-24, Eindhoven, The Netherlands, 2009)

17. AMPLE: Aspect-Oriented, Model-Driven, Product Line Engineering [http://cordis.europa.eu/search/index.cfm?fuseaction=proj.document\& amp;PJ_RCN=8724342]

18. H Wada, EMM Babu, A Malinowski, J Suzuki, K Oba, in IASTED Int'l Conf. on Software Engineering and Applications. Design and implementation of the matilda distributed UML virtual machine, (Dallas, TX, USA), p. 2006

19. K Czarnecki, U Eisenecker, in Proceedings of the European Software Engineering Conference. Components and generative programming, (Toulouse, France, 1999), pp. 2-19

20. IDA: About. [http://www.hex-rays.com/products/ida/index.shtml]

21. K Kang, S Cohen, J Hess, W Nowak, S Peterson, Feature-Oriented Domain Analysis (FODA) Feasibility Study, (CMU/SEI-90-TR-21). (Software Engineering Institute, Carnegie Mellon University, Pittsburgh, PA, 1990). [http://www.sei.cmu.edu/library/abstracts/reports/90tr021.cfm]

22. V Cechticky, P Chevalley, A Pasetti, W Schaufelberger, in GPCE'03 Proceedings of the 2nd international conference on Generative programming and component engineering. A generative approach to framework instantiation, (Erfurt, Germany, 2003), pp. 267-286

23. K Czarnecki, M Antkiewicz, in ACM SIGSOFT/SIGPLAN International Conference on Generative Programming and Component Engineering (GPCE'05). Mapping features to models: a template approach based on superimposed variants, (Tallinn, Estonia, 2005), pp. 422-437

24. A Gurtovoy, D Abrahams, The boost MPL library. [http://www.boost.org/ doc/libs/1_48_0/libs/mpl/doc/index.html]

25. D Abrahams, A Gurtovoy, C++ Template Metaprogramming: Concepts, Tools, and Techniques from Boost and Beyond. (Addison-Wesley Professional, Boston, 2004)

\section{doi:10.1186/1687-3963-2012-7}

Cite this article as: Cardoso et al: A generative-oriented model-driven design environment for customizable video surveillance systems. EURASIP Journal on Embedded Systems 2012 2012:7. 